\title{
The electroviscous effect for a confined nanosphere in solution
}

\author{
Ali Behjatian, Maria Bespalova, Narain Karedla, and Madhavi Krishnan* \\ Physical \& Theoretical Chemistry Laboratory, Department of Chemistry, \\ South Parks Road, University of Oxford, Oxford OX1 3QZ, United Kingdom
}

\begin{abstract}
A charged colloidal particle suspended in an electrolyte experiences electroviscous stresses arising from motion-driven electrohydrodynamic phenomena. Under certain conditions, the additional contribution from electroviscous drag forces to the total drag experienced by the moving particle can lead to measurable deviations of particle diffusion coefficients from values predicted by the well known Stokes-Einstein relation that describes diffusive behavior of small particles in an unbounded charge-free fluid. In this study, we investigate the role of electroviscous stresses on nanoparticle diffusion in confined geometries using both simulations and experiment. We compare our experimental measurements with the results of a numerically solved continuum model based on the PoissonNernst-Planck-Stokes system of equations and find good agreement between experiment and theory. Depending on the radius of the counterion species in solution and the degree of confinement, we find that the viscous drag on polystyrene nanoparticles can be augmented by approximately 10-25\% compared to the values predicted by pure hydrodynamic models in the absence of free charge in the fluid. This enhancement corresponds approximately to a $5-10 \%$ increase compared to the electroviscous contribution for a charged particle in an unbounded fluid. Contrary to recent reports in the experimental literature, we find neither experimental nor theoretical evidence of an anomalously large enhancement of electroviscous forces on a confined charged nanoparticle in solution.
\end{abstract}

Keywords: Electroviscous effect; Brownian motion; Electrohydrodynamics; Electrokinetic phenomena

\section{INTRODUCTION}

Transport of colloidal particles and macromolecules in confined geometries is of great interest in a wide range of biological [1-4], physical and engineering contexts [58]. For a particle undergoing random thermal motion in an unbounded fluid, the diffusion coefficient is given by the well-known Stokes-Einstein relation, $D_{\infty}=k_{\mathrm{B}} T / \gamma$, where $k_{\mathrm{B}}$ and $T$ represent Boltzmann constant and temperature, respectively. Here, $\gamma=6 \pi \mu a$ is a friction coefficient derived from Stokes' law which gives the drag force exerted on a spherical particle of radius, $a$, freely moving in a fluid of viscosity, $\mu$. Viscous forces on a moving object derive from long-ranged hydrodynamic flow fields that can be strongly modified by the presence of boundaries. The problem of calculating the viscous retardation due to the presence of hydrodynamic boundaries in the vicinity of a moving object has received substantial theoretical attention [9-15]. These studies have shown that the friction coefficient $\gamma$ is indeed modified due to the presence of boundaries, and the diffusion coefficient of a confined particle, which is a common experimental measurable, can turn anisotropic and particle-geometry dependent [16]. Experiments on micro- and nanoparticles confined in parallel-plate slits or near a single wall have tested these models and have demonstrated that the measurements are in good agreement with theory, with the measurements in some cases lying within $5 \%$ of the theoretical prediction [17-20].

The case of a charged diffusing particle adds an important and interesting dimension to the general problem of

\footnotetext{
*madhavi.krishnan@chem.ox.ac.uk
}

confined diffusion, and is the focus of this study. Colloidal particles or molecules in the fluid phase generally acquire net electrical charge on account of associative or dissociative reactions of chemical groups they carry [21]. Screening of the electrical potential at the surface by a cloud of oppositely charged counterions in solution creates an electric double layer (EDL) around the particle whose thickness is of the order of the Debye screening length $\kappa^{-1}=\sqrt{\epsilon_{0} \epsilon_{\mathrm{r}} k_{\mathrm{B}} T / \sum z_{i}^{2} n_{i}^{0} e^{2}}$. Here, $e, \epsilon_{\mathrm{r}}, \epsilon_{0}, n_{i}^{0}$ and $z_{i}$ are the elementary charge, relative permittivity of the aqueous medium, permittivity of free space, bulk concentration and valence of the $i$ th ionic species, respectively. Perturbation of the equilibrium distribution of ions in the EDL by an external electrical or flow field, or indeed due to thermal motion of the particle, can lead to additional forces on the particle, modifying the overall drag force. The phenomenon has been termed the electroviscous effect and has been examined theoretically for particles suspended in a bulk fluid [22-26].

For a slowly moving particle in an unbounded fluid the equilibrium distribution of ions is perturbed by the flow induced by the motion of the particle, but the counterion cloud can still follow the moving object through the fluid. At steady state, the particle together with its double layer form a system which is electrically equivalent to an open circuit; i.e., there is zero net electric current in the particle and its double layer at steady state. This implies that for small distortions, an electric field must be induced such that its conductive current cancels the current due to convection of charges by the fluid within the EDL. The induced electric field can affect the motion of particle in two ways: first, the field can act on the charge carried by particle, and second, the field induces an electroosmotic flow by acting on the mobile ions in the fluid. These mechanisms enhance the viscous drag by in- 
troducing an additional force known as the electroviscous force, which is proportional to velocity of the particle in the regime of small Péclet numbers where the distortions are negligible. The effect of electrokinetic phenomena on the motion of small particles in free solutions has been investigated in numerous studies $[23,24,27,28]$. Ohshima et. al [24] examined the impact of charges on the sedimentation of charged colloidal particles in an electrolyte. Using a semi-analytical approach to solve the coupled linearized Poisson-Nernst-Planck and Stokes (PNPS) equations for arbitrary EDL thickness and surface potentials, the analysis suggested that the terminal velocity of the particle can be reduced by a maximum of $8 \%$ when the EDL thickness is of the order of one particle radius; i.e. $\kappa a=1$. In other words, the study revealed that the contribution of electrostatics to the overall drag is maximal when the particle size is comparable to the spatial extent of the counterion cloud. Typically, the thickness of the neutralizing cloud of ions in aqueous systems is in the range of 1-40 nm which implies that the electroviscous effect is important for nanometer scale particles where the double layer thickness, $\kappa^{-1}$ is comparable to the size of particle, $a$.

A charged colloidal particle undergoing Brownian motion performs successive jumps due to random collisions with solvent molecules. The characteristic time of a jump is the momentum relaxation timescale, given by $\tau=m_{\mathrm{p}} / \gamma$, where $m_{\mathrm{p}}$ is the mass of particle and $\gamma$ is the associated friction coefficient [29]. This time-scale $\tau$ is the time required for a particle to be brought to rest by the viscous force acting on it after it has received a random kick from the solvent. A natural velocity scale for the Brownian motion of a particle can be obtained by assuming thermal equilibrium between the particle and the surrounding fluid. Following this assumption, the velocity distribution of the particle will be Maxwellian [30], and the mean kinetic energy of particle, $E_{\text {kin }}$, will be described by a fixed quantity which is a function of the temperature, $T$, of the fluid [31],

$$
E_{\text {kin }}=\frac{1}{2} m_{\mathrm{p}}\langle\mathbf{U} \cdot \mathbf{U}\rangle=\frac{3}{2} k_{\mathrm{B}} T,
$$

where $\mathbf{U}$ is the velocity of the particle. The above equation indicates that $u_{\mathrm{s}}=\sqrt{k_{\mathrm{B}} T / m_{\mathrm{p}}}$ is an appropriate velocity scale of the problem which defines the characteristic length of a jump by $l_{\mathrm{s}}=\tau u_{\mathrm{s}}$. The relaxation time of the ions in solution after each jump of the particle, given by $\tau_{\mathrm{r}}=l_{\mathrm{s}}^{2} / D_{\text {ion }}$, is another relevant time-scale of the problem. Here, $D_{\text {ion }}$ is the diffusion coefficient of the ions which can be written in terms of the radius of ions, $r_{\text {ion }}$, by applying Stokes-Einstein relation; i.e. $D_{\text {ion }}=k_{\mathrm{B}} T / 6 \pi \mu r_{\text {ion }}$. It is evident that the EDL can retain its equilibrium spatial distribution during these consecutive jumps provided $\tau_{\mathrm{r}} \ll \tau$ [32]. For a spherical particle of radius $a$ with $\gamma$ being $6 \pi \mu a$, this condition reduces to $r_{\text {ion }} \ll a$, which is satisfied for particles of radii of the order of $10 \mathrm{~nm}$. Motivated by the above scaling analysis, Schumacher and van de Van [32] used
Ohshima's theory to examine the impact of electrokinetic forces on Brownian motion of charged particles observed in experiments. They measured the diffusion coefficient of gold sols and latex particles for a wide range of $\kappa a$ values and observed a minimum in the diffusion coefficient for $\kappa a \approx 1$. It was also observed that the minimum values were $3-8 \%$ lower than those measured in high $(\kappa a \gg 1)$ or low ionic $(\kappa a \ll 1)$ concentrations. This observation was in agreement with the theoretical result of Ref. [24] that predicts electroviscous effect vanishes at both extremes of $\kappa a$ range $(\kappa a \gg 1$ and $\kappa a \ll 1)$.

However, several recent experimental measurements of diffusion coefficients for particles confined in low ionic strength aqueous solution have reported anomalously low values which cannot be explained on the basis of pure hydrodynamics. For example, Eichmann et al. [33] reported diffusion coefficients that were $30-50 \%$ smaller than those given by the Stokes-Einstein relation including hydrodynamic corrections for the presence of boundaries in the vicinity of the particle. A subsequent study [18] demonstrated that the effect vanishes when all surfaces are coated with proteins that mask the surface charge in the system, indicating that the anomalously small diffusion coefficients were due to the presence of charged surfaces in the system. The observed drag enhancement was attributed to a large electroviscous contribution due to confinement. Other studies have reported mixed results under slightly different conditions. In one instance, Kaji et al. [34] have observed a reduction in the diffusion coefficient of nano-sized polystyrene carboxylated particles by a factor of approximately 3 and 5 in slit-like channels, and channels in the presence of nano-pillars, respectively. They attributed this effect to enhancement of water viscosity under confinement. Similar observations of apparent drag enhancement have been reported for confined quantum dots and for gold nanoparticles confined in electrostatic fluidic traps [35, 36]. Recent measurements on gold nanospheres confined between a glass surface and a polymer-coated surface revealed a strong monotonic decay of the diffusion coefficient with decreasing gap distance between surfaces, and the values were found to be 20-50\% lower than the predictions based on pure hydrodynamic theories [37]. On the other hand, measurements by Kazoe et al. [38] on charged polystyrene beads in nanochannels under comparable conditions demonstrated that contrary to previous studies, measured diffusion coefficients were in fact in agreement with the Stokes-Einstein equation including hydrodynamic corrections for wall effects. Furthermore, there have been suggestions that the anomalous retardation reported in Ref. [33] could be influenced by transient adsorption of the particles to the slit walls. Overall it remains unclear as to whether confinement of charged particles can indeed result in anomalously large drag enhancements in experiment, much above that expected in free solution, and if such behavior can be rationalized using existing theoretical models. The results of these experimental measurements are summarized in Table I. Un- 
surprisingly, the measured diffusion coefficients, $D$, are smaller than the bulk values, $D_{\infty}$, however in nearly all cases, corrected diffusion coefficients, $D_{\mathrm{h}}$, suggested by purely hydrodynamic theories do not fully explain the disparity in the measurement; i.e $D_{\mathrm{h}} / D>1$. Considering the Stokes-Einstein relation, this implies that the total drag force in confinement is perhaps affected by an additional resistive force which originates from sources different than pure hydrodynamic interactions between particles and wall, potentially including a contribution from mobile charge in the fluid.

TABLE I. Summary of the diffusion measurements in experimental studies involving various types of charged particle confined between silica walls. Here, $D_{\mathrm{h}}$ represents the diffusion coefficients corrected for hydrodynamic interactions between the particle and confining walls.

\begin{tabular}{|c|c|c|c|c|}
\hline Study & $\overline{a(\mathrm{~nm})}$ & $2 h(\mathrm{~nm})$ & $D_{\infty} / D$ & $\overline{D_{\mathrm{h}} / D}$ \\
\hline \multirow[t]{3}{*}{ 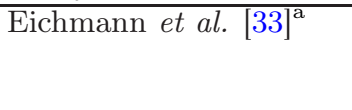 } & 28.5 & 342 & 2.65 & 2.21 \\
\hline & 61.5 & 342 & 2.18 & 1.42 \\
\hline & 125 & 619 & 3.02 & 2.02 \\
\hline Hoang et al. $[35]^{b}$ & 12.5 & 150 & $\approx 3$ & 2.40 \\
\hline Kaji et al. $[34]^{\mathrm{C}}$ & 25 & 400 & $\overline{3.11}$ & $\overline{2.72}$ \\
\hline Mojarad\&Krishnan [36] & 40 & 215 & 3.40 & 2.21 \\
\hline Eichmann\&Bevan $[18]^{\mathrm{e}}$ & 64 & 269 & 1.47 & $\overline{0.86}$ \\
\hline Kazoe et al. $[38]^{\mathrm{f}}$ & 32 & 410 & 1.29 & 1.10 \\
\hline \multirow{6}{*}{\multicolumn{5}{|c|}{$\begin{array}{l}\text { a Gold nanoparticles in a solution of } 0.1 \mathrm{mM} \text { Sodium Dodecyl } \\
\text { Sulfate (SDS) corresponding to a Debye length of } \kappa^{-1}=30 \mathrm{~nm} \\
\text { b Quantum dots in nanoslits. The Debye length is not reported } \\
\text { but it is estimated that } \kappa a \ll 1 \text {. } \\
\text { c Polystyrene nanospheres in nanoslits filled with water. } \\
\text { d } \text { Gold nanoparticles in electrostatic fluidic traps. } \\
\text { e Experiments are done under conditions comparable to Ref. } \\
\text { [33], however the surfaces are coated by proteins in order to } \\
\text { eliminate electroviscous contributions. }\end{array}$}} \\
\hline & & & & \\
\hline & & & & \\
\hline & & & & \\
\hline & & & & \\
\hline & & & & \\
\hline \multicolumn{5}{|c|}{$\begin{array}{l}{ }^{\mathrm{f}} \text { Polystyrene particles in nanoslits filled with a solution of } \\
\mathrm{Na}_{2} \mathrm{~B}_{4} \mathrm{O}_{7} \text {. }\end{array}$} \\
\hline
\end{tabular}

In the present study, we examine the effect of electroviscous stresses on the diffusive behavior of charged colloidal nanoparticles under confinement. To this end, using a numerical model based on PNPS system of equations, we first evaluate the combined effect of confinement and electroviscous forces on the total drag exerted on a charged particle in motion. Owing to the linear behavior of electroviscous forces at low $P e$ regime, the presence of charges in the system leads to modification of the friction coefficient, $\gamma$. We employ the fluctuation-dissipation theorem [39] to relate the calculated drag force to the diffusion coefficient using a modified Stokes-Einstein relation. This approach has been utilized extensively to estimate the diffusion coefficient of particles under different conditions such as diffusion near a single wall [19] or between two parallel plates $[17,20,40]$. Note that due to complexities of the PNPS system of equations, performing more sophisticated simulations such as Brownian Dynamics (BD) simulations including electrohydrodynamics is extremely costly and practically infeasible. Hence, in this study, we attempt to address the problem by utilizing the fluctuation-dissipation theorem which leads to a modified Stokes-Einstein relation. We then examine the impact of electroviscous stresses on a confined nanoparticle in solution by experimentally measuring the diffusion coefficient of polystyrene particles in parallel-plate nanofluidic silica slits of different depths, and in the presence of different counterion species in solution. The goal of the study is to explore the possibility of anomalously hindered diffusion due to electroviscous effects by making a controlled comparison of experimentally measured drag coefficients with corresponding theoretical calculations.

\section{PROBLEM DESCRIPTION}

The effect of confining boundaries on the viscous drag exerted on a sphere moving with a constant velocity, $U$, in a pure fluid devoid of electrical charge, has been examined in numerous theoretical works. For a detailed summary of these studies, we refer the reader to Ref. [41] which provides solutions to a large number of relevant problems. Among them, two problems are particularly attractive for the purpose of this study, namely the drag on a sphere traveling along the axis of a pipe, and drag exerted on a spherical particle moving at the mid-plane of two infinitely large flat plates. Both of these problems were initially addressed by Faxén [9] using the method of reflection, however, Bohlin [42] found a more accurate solution for the former case by extending Faxén's method to obtain a higher order solution. The solutions show that, in general, the hydrodynamic force, $F_{\mathrm{h}}$, can be expressed as $F_{\mathrm{h}}=K F_{\infty}$, where $K>1$ is a geometric factor which leads to augmentation of the drag force in comparison with that in unbounded domain given by Stokes' law $F_{\infty}=6 \pi \mu a U$. Here, $\mu$ is the viscosity of fluid and $a$ is the radius of sphere. The geometric factors for the above problems are described by

$$
\begin{array}{r}
K_{\text {slit }}=\left[1-1.004\left(\frac{a}{h}\right)+0.418\left(\frac{a}{h}\right)^{3}+\right. \\
\left.0.21\left(\frac{a}{h}\right)^{4}-0.169\left(\frac{a}{h}\right)^{5}\right]^{-1},
\end{array}
$$

and

$$
\begin{array}{r}
K_{\text {pipe }}=\left[1-2.10443\left(\frac{a}{R}\right)+2.08877\left(\frac{a}{R}\right)^{3}-\right. \\
0.94813\left(\frac{a}{R}\right)^{5}-1.372\left(\frac{a}{R}\right)^{6}+ \\
\left.3.87\left(\frac{a}{R}\right)^{8}-4.19\left(\frac{a}{R}\right)^{10}\right]^{-1},
\end{array}
$$

where $2 h$ and $R$ represent the distance between plates and radius of the pipe, respectively. Here, our main goal is to extend the discussed problems by considering the contribution of electrokinetic phenomena in order to evaluate the additional drag due to electroviscous stresses. In order to gain some preliminary insight into the problem, 


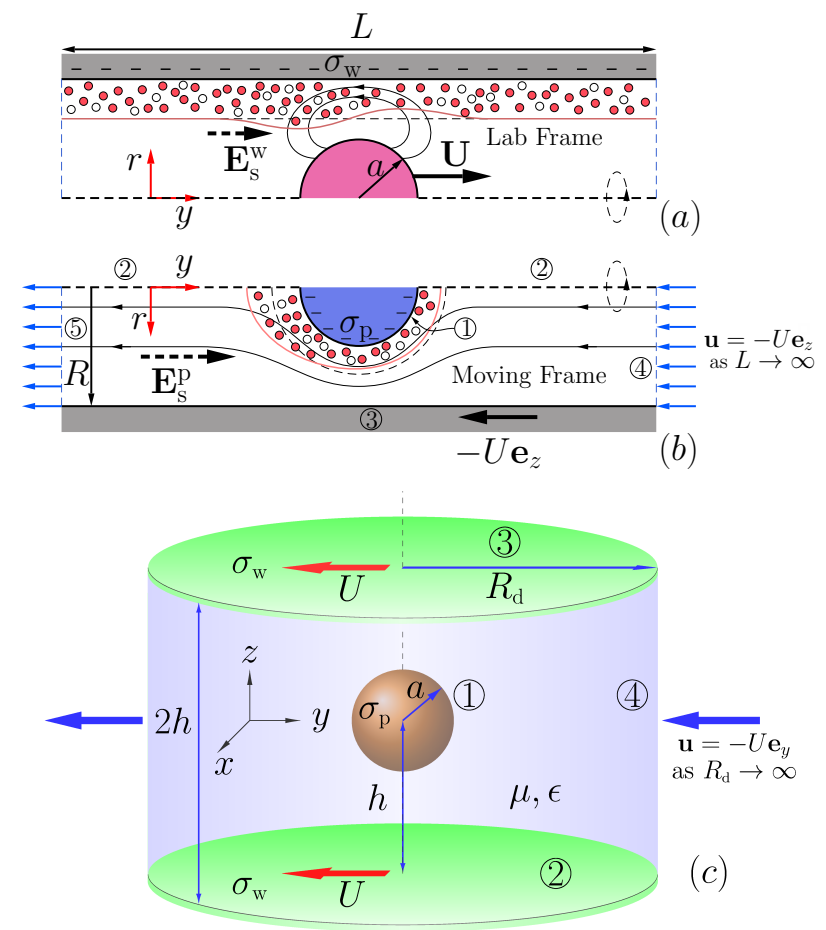

FIG. 1. Schematic depiction of the confined nanosphere geometry used for computation. (a) Axisymmetric set-up for an uncharged particle moving along the axis of a circular pipe depicting the distortion of the double layer in the vicinity of a charged wall in the laboratory frame of reference. (b) Deformation of the counterion cloud around the particle due to its motion along the axis of an uncharged pipe, in the particle frame of reference. Closed and open circles represent positive and negative ions in the EDLs, respectively. (c) 3D domain for a particle at the midplane of a slit created by parallel plates separated by a distance of $2 h$.

we first consider a spherical particle of radius, $a$, confined in a tube of radius, $R$, to explain the mechanisms which give rise to the electroviscous drag. The particle moves with a constant velocity $U$, and the surface of the tube carries a uniform negative electrical surface charge. For rectilinear motion of the particle along the axis of an infinitely long pipe, the fluid leaks through the gap between the particle and wall. Figure 1a depicts the streamlines in the laboratory frame of reference attached to the wall. As shown in the figure, the flow in the gap would be expected to displace ions in the EDL towards the tail of the particle and induce a convective current within the EDL. This convective current must be canceled by conductive current due to $\mathbf{E}_{\mathrm{s}}^{\mathrm{w}}$ at the steady state. Furthermore, the electroosmotic flow arising from the induced electric field $\mathbf{E}_{\mathrm{s}}^{\mathrm{w}}$ would be in the direction opposite to the flow in the gap. This additional flow opposes free leakage of fluid through the gap and thereby imposes an additional drag on the particle. All these features point qualitatively in the direction of an enhanced electroviscous drag on a charged confined particle in solution [43-45]. It should be noted that in the above de- scription, we assumed that the fluid only squeezes around the particle, and the movement of particle does not produce long-range non-vanishing velocity fields which has been recently reported for particles tightly confined in pipes with open ends [46]. Misiunas et al. [46] have demonstrated long-range interactions between two confined particles in tubes with open ends, and have shown that this phenomenon arises from long-range hydrodynamic interactions as a result of a non-vanishing axial flow along the pipe. Their observations showed that an axially moving particle is also capable of driving the fluid along the pipe to induce a non-vanishing flow in axial direction. This axial flow reduces to a simple Poiseuille flow far away from particle, and they provided an approximation of the mean fluid velocity associated with this Poiseuille flow, $\langle u\rangle$, for a sphere of radius, $a$, moving with velocity, $U$, along the axis of a tube of radius, $R$, and length, $L$, and have shown that

$$
\frac{\langle u\rangle}{U}=\frac{R^{3} a}{R^{3} a+L \delta^{5 / 2} a^{1 / 2}},
$$

where $\delta=R-a$ is the minimum gap distance between particle and wall. The above equation can be rewritten as

$$
\frac{\langle u\rangle}{U}=\frac{a / L}{a / L+\sqrt{\frac{a}{R}\left(1-\frac{a}{R}\right)^{5}}},
$$

which implies $\langle u\rangle / U$ is negligible provided $a / L \ll$ $\sqrt{(a / R)(1-a / R)^{5}}$. For a highly confined case of say $a / R=0.5$, the above approximation reveals that $\langle u\rangle / U \approx 2 \%$ if $a / L=0.0025$. This condition is typically met for a wide range of nanochannels where the length of the channel is of the order of a millimeter but the height or diameter is of the order of hundreds of nanometers. Note that since particle motion can only generate a finite pressure gradient, a moving particle cannot displace a liquid column of infinite length. Hence, provided that the length of the pipe is considerably larger than its radius, the long-range Poiseuille flow due to the perturbation of particle will be negligible [31, 46]. This allows one to assume that the fluid is nearly at rest far away form particle, and simplifies the boundary conditions which will be discussed in the next section.

Turning our attention to the EDL around the particle, it is more appropriate to switch to a frame of reference moving with the particle. Figure $1 \mathrm{~b}$ depicts the streamlines in this frame of reference, and illustrates the distorted cloud of counterions around the particle in a situation where the wall carries no charge. Here, an electric field, $\mathbf{E}_{\mathrm{s}}^{\mathrm{p}}$, must be induced such that its conductive current cancels the convective current at the steady state. Therefore, the induced electric field leads to a higher drag by retarding the electrically charged particle directly, as well as by generating an electroosmotic flow by acting upon mobile ions in the fluid. It is worth mentioning that in the literature, the phenomenon described above 
is known as the primary electroviscous effect. In other words, the primary effect is concerned solely with the resistance to motion arising from distortions of the counterion clouds in the system. In concentrated suspensions, repulsions between the particles give rise to a secondary effect which is vanishingly small at low particle volume fractions. For macromolecules such as proteins, there also exists a tertiary effect which is attributed to conformational and structural changes arising from intraparticle interactions [47]. Since the present study as well as other studies discussed in Sec. I are restricted to very low volume fractions and single particle measurements in dilute systems of particles, the secondary effect is very small and negligible. In the following section, we present our model for a single charged confines particle in detail and develop on the results in subsequent sections.

\section{GOVERNING EQUATIONS AND DIMENSIONLESS PARAMETERS}

The equations describing electrohydrodynamic behavior in our system are the incompressible Navierstokes (NS) and continuity equations, the steady state convection-electrodiffusion equation for the charged ionic species in solution, and Poisson equation which represents the reduced form of Maxwell's equations when magnetic fields are negligible [27]. Neglecting the inertia term in the NS equation in the limit of low Reynolds numbers, $R e=\rho U a / \mu$, where $U$ is the particle velocity, we solve the Stokes equation for the fluid flow. The governing equations are therefore:

$$
\begin{gathered}
\mu \nabla^{2} \mathbf{u}-\nabla p-\rho_{\mathrm{e}} \nabla \phi=0, \\
\nabla \cdot \mathbf{u}=0 \\
-\nabla \cdot(\epsilon \nabla \phi)=\rho_{\mathrm{e}} \\
\nabla \cdot \mathbf{J}_{i}=0
\end{gathered}
$$

where $\mathbf{u}, p, \epsilon=\epsilon_{0} \epsilon_{\mathrm{r}}, \rho_{\mathrm{e}}=\sum z_{i} e n_{i}$ and $\phi$ are the velocity of the fluid, pressure, permittivity of aqueous medium, charge density and electric potential, respectively. Here, the ionic flux $\mathbf{J}_{i}$ is defined as

$$
\mathbf{J}_{i}=-D_{i} \nabla n_{i}+n_{i} \mathbf{u}-\frac{D_{i} z_{i} e}{k_{\mathrm{B}} T} n_{i} \nabla \phi
$$

where $n_{i}$ is the number density of the $i$ th ionic species and $D_{i}$ represents its corresponding diffusion coefficient. For the case of symmetric-monovalent electrolytes $\left(z_{1}=\right.$ $-z_{2}=1$ ), we replace the index $(i)$ with $(+)$ and $(-)$ to distinguish the positive and negative ions, respectively. Table II provides the values of diffusion coefficients for ionic species used in subsequent simulations.
TABLE II. Diffusion coefficients and stokes radii for different ionic species used in the simulations.

\begin{tabular}{lcc}
\hline \hline Ion & $D_{\text {ion }}\left(\mathrm{m}^{2} \mathrm{~s}^{-1}\right)$ & $r_{\text {ion }}(\AA)$ \\
\hline $\mathrm{K}^{+}$ & $1.957 \times 10^{-9 \mathrm{~b}}$ & $1.25^{\mathrm{a}}$ \\
$\mathrm{Cs}^{+}$ & $2.056 \times 10^{-9 \mathrm{~b}}$ & $1.19^{\mathrm{a}}$ \\
$\mathrm{Li}^{+}$ & $1.029 \times 10^{-9 \mathrm{~b}}$ & $2.39^{\mathrm{a}}$ \\
$\mathrm{THA}^{+}$ & $0.413 \times 10^{-9 \mathrm{c}}$ & $5.94^{\mathrm{c}}$ \\
$\mathrm{Cl}^{-}$ & $2.032 \times 10^{-9 \mathrm{~b}}$ & $1.21^{\mathrm{a}}$ \\
\hline
\end{tabular}

a Stokes radius based on stick condition [48].

b Diffusion coefficients are calculated form molar conductivity of ions at infinite dilution [49].

${ }^{c}$ Values are obtained by extrapolation of data from Ref. [49] which provides diffusion coefficients for lighter tetra-alkyl-ammonium ions.

TABLE III. A summary of the boundary conditions used for the numerical simulations.

\begin{tabular}{cccc}
\hline \hline Boundary & Poisson & Nernst-Planck & Stokes \\
\hline \multicolumn{5}{c}{ Axisymmetric Problem } \\
\hline (1) & $-\epsilon \nabla \phi \cdot \mathbf{n}=\sigma_{\mathrm{p}}$ & $\mathbf{J}_{ \pm} \cdot \mathbf{n}=0$ & $\mathbf{u}=\mathbf{0}$ \\
(2) & Symmetry & Symmetry & Symmetry \\
(3) & $-\epsilon \nabla \phi \cdot \mathbf{n}=\sigma_{\mathrm{w}}$ & $\mathbf{J}_{ \pm} \cdot \mathbf{n}=0$ & $\mathbf{u}=-U \mathbf{e}_{y}$ \\
(4) & $-\epsilon \nabla \phi \cdot \mathbf{n}=0$ & $n_{ \pm}=n_{ \pm}^{\mathrm{eq}}$ & $\mathbf{u}=-U \mathbf{e}_{y}$ \\
(5) & $-\epsilon \nabla \phi \cdot \mathbf{n}=0$ & $n_{ \pm}=n_{ \pm}^{\mathrm{eq}}$ & $\mathbf{u}=-U \mathbf{e}_{y}$ \\
\hline \multicolumn{5}{c}{$3 \mathrm{D}$-slit Problem } \\
\hline (1) & $-\epsilon \nabla \phi \cdot \mathbf{n}=\sigma_{\mathrm{p}}$ & $\mathbf{J}_{ \pm} \cdot \mathbf{n}=0$ & $\mathbf{u}=\mathbf{0}$ \\
(2) & $-\epsilon \nabla \phi \cdot \mathbf{n}=\sigma_{\mathrm{w}}$ & $\mathbf{J}_{ \pm} \cdot \mathbf{n}=0$ & $\mathbf{u}=-U \mathbf{e}_{y}$ \\
(3) & $-\epsilon \nabla \phi \cdot \mathbf{n}=\sigma_{\mathrm{w}}$ & $\mathbf{J}_{ \pm} \cdot \mathbf{n}=0$ & $\mathbf{u}=-U \mathbf{e}_{y}$ \\
(4) & $-\epsilon \nabla \phi \cdot \mathbf{n}=0$ & $n_{ \pm}=n_{ \pm}^{\mathrm{eq}}$ & $\mathbf{u}=-U \mathbf{e}_{y}$ \\
\hline
\end{tabular}

The species listed in the table correspond to 4 monovalent salts, namely $\mathrm{KCl}, \mathrm{CsCl}, \mathrm{LiCl}$, and Tetrahexylammonium Chloride (THAC).

A no-slip boundary condition for fluid velocity is applied at all solid surfaces. In the frame of reference of the particle, this implies a zero velocity at the surface of particle and a uniform velocity of $-U$ in the $y$-direction at the walls of pipe or slit (Fig. 1). Similarly, at the outer boundaries of the pipe and slit, where the perturbation of the sphere vanishes as $L \rightarrow \infty\left(R_{\mathrm{d}} \rightarrow \infty\right)$, we use a uniform velocity in the $y$-direction as the boundary condition. A constant surface charge density is applied at all solid surfaces and is denoted by $\sigma_{\mathrm{p}}$ at the particle surface and $\sigma_{\mathrm{w}}$ at the wall. We assume that the electrical fields induced by particle motion vanish far away from the particle, implying that at a large distance from the particle the spatial potential distribution in the slit is identical to that of a particle-free, electrolyte-filled slit at equilibrium; i.e., $\nabla \phi \cdot \mathbf{n}=0$ at the edges of the geometry. Furthermore, we assume that matter cannot penetrate any surface and therefore employ a no-flux boundary condition for Eq. (9) at the particle surface and slit walls. The bulk concentrations for both ionic species are assumed to be equal for all the systems under consideration; i.e., $n_{+}^{0}=n_{-}^{0}=n_{\mathrm{b}}$. The concentrations of ionic species at 
the edges of the slit and pipe domains are held constant at their equilibrium values, $n_{ \pm}^{\mathrm{eq}}=n_{\mathrm{b}} \exp \left(\mp \phi^{\mathrm{eq}} e / k_{\mathrm{B}} T\right)$, where $\phi^{\text {eq }}$ is the electrical potential when the particle is stationary; i.e. $U=0$. This equilibrium potential, $\phi^{\mathrm{eq}}$, is obtained from the solution of the Poisson-Boltzmann equation for the corresponding system. Table III summarizes these boundary conditions for the axisymmetric and 3D slit problems shown in Fig. 1. Since the Poisson equation (8) with a pure Neumann boundary condition is undetermined up to a constant, a constraint must be applied to the potential, $\phi$, in order to obtain a unique solution. This constraint can be applied either by fixing the potential at a single point in the domain or by constraining the volume average of the potential [50]. We adopt the second approach and constrain the average of potential to the average of the equilibrium potential $\phi^{\text {eq; }}$ i.e. $\int_{V} \phi d v=\int_{V} \phi^{\mathrm{eq}} d v$.

The dimensionless parameters of interest in the system are $P e=U a / D_{+}, \omega=D_{+} / D_{-}, S_{\mathrm{p}}=\sigma_{\mathrm{p}} e a / \epsilon k_{\mathrm{B}} T$, $S_{\mathrm{w}}=\sigma_{\mathrm{w}} e a / \epsilon k_{\mathrm{B}} T, H a=n_{\mathrm{b}} k_{\mathrm{B}} T a / \mu U$ and $s=\kappa a$. Here $P e$ is the ratio of diffusion time scale of ions in the system, $a^{2} / D_{+}$, to the time scale of convection, $a / U$, and $s$ is a dimensionless system size. Further, $\omega$ is the ratio of the diffusion coefficients of the ionic species in solution, while $S_{\mathrm{p}}$ and $S_{\mathrm{w}}$ represent the dimensionless surface charge density of the particle, $\sigma_{\mathrm{p}}$, and the wall, $\sigma_{\mathrm{w}}$, respectively. Finally, the electric Hartmann number, $\mathrm{Ha}$, gives the ratio of electric forces to viscous forces in the system. For motion driven electrokinetic phenomena, Yariv et. al [51] demonstrated that $\mathrm{Ha}$ can be written in terms of $s, P e$, and another dimensionless parameter $\alpha=\epsilon \zeta_{\mathrm{T}}^{2} / \mu D_{+}$; i.e., $H a=s^{2} P e^{-1} \alpha$. Here, $\zeta_{\mathrm{T}}=k_{\mathrm{B}} T / e$ is the thermal potential, and therefore, $\alpha$ is a property of the electrolyte independent of the geometry of the system. For typical aqueous ionic solutions, this parameter is of order of unity ( $\alpha \approx 0.5$ ), and hence for most cases, the magnitude of $\mathrm{Ha}$ is determined by the other two dimensionless parameters of the system namely, $\mathrm{Pe}$ and $\mathrm{s}$. In other words, $\mathrm{Ha}$ is not an independent non-dimensional parameter once the properties of electrolyte are fixed; i.e., $\alpha$ becomes a fixed parameter for the system.

\section{IMPLEMENTATION AND VALIDATION OF THE THEORETICAL MODEL}

In order to compute the contribution of electroviscous stresses to the total drag force, $\mathbf{F}_{\mathrm{t}}$, acting on a particle in a confined system, we numerically solved the above system of equations subject to the relevant boundary conditions. As shown in Fig. 1, we consider two different geometries in the computation. The first is a simplified 2D axisymmetric setup corresponding to a particle of radius, $a$, moving along the axis of a pipe of radius, $R$ (Fig. 1b), and the second is a three-dimensional parallel-plate slit geometry corresponding to the experiments consisted of a particle of radius, $a$, confined between two parallel disks separated by a distance, $2 h$ (Fig. 1c). The computational cost of calculations in a $2 \mathrm{D}$ axisymmetric geometry is much lower than that of a 3D system, so we examine general trends in the axisymmetric case and perform 3D computations only for comparison with experiments and specific cases of interest.

The confined particle system is assumed to be in equilibrium with an electrolyte reservoir where the bulk concentration of ionic species is given by $n_{\mathrm{b}}$. Our investigations are in the regime of slightly overlapping double layers $(\kappa h=4.3,6.6)$, where the most probable location of the confined particle is, in practice, Boltzmann-weighted to the mid-plane of slit. This inherent geometrical symmetry may be exploited to reduce computational cost, and therefore in our geometric models for computation the center of the sphere is always located at the mid-plane of the slit or axis of the cylindrical pipe.

We applied Finite Element (FE) analysis to solve the nonlinear system of Eqs. (6-9), numerically. We used COMSOL Multiphysics for the axisymmetric cases and FEniCS [52] which is an open-source software for the three-dimensional slit geometry. For axisymmetric cases with a few thousands of degrees of freedom, the discretized equations can be solved efficiently by direct solvers using a fully coupled numerical scheme, however, a hybrid scheme using iterative solvers was found to be more effective for large 3D systems involving millions of degrees of freedom. In this scheme, Eqs. (8) and (9) are discretized together using a coupled block solver and Eq. (6) is solved subsequently using a fixed point iteration technique. Further details may be found in Ref. [53].

We computed the spatial distribution of electrical potential and the hydrodynamic flow field in the system for a particle moving at constant velocity, $U \propto u_{\mathrm{s}}$. We then determined the total drag force exerted on the particle by integration of the traction vector, $\mathbf{t}=\mathbf{T} \cdot \mathbf{n}$, over the surface of particle $S$; i.e., $\mathbf{F}_{\mathrm{t}}=\int_{S} \mathbf{t} d s$. Here, $\mathbf{n}$ is the unit outward normal vector at the surface of particle and $\mathbf{T}=\mathbf{T}^{\mathrm{h}}+\mathbf{T}^{\mathrm{e}}$ is the total stress tensor, where $\mathbf{T}^{\mathrm{h}}$ and $\mathbf{T}^{\mathrm{e}}$ represent the hydrodynamic [54] and Maxwell stress [55] tensors, respectively. In practice due to the symmetries, only $y$-component of the total force, $F_{\mathrm{t}}^{y}$, is non-zero, and therefore, we use the magnitude of this component, $F_{\mathrm{t}}=\left|F_{\mathrm{t}}^{y}\right|$, for our subsequent analyses.

The role of electroviscous stresses on the sedimentation of a charged colloidal particle was studied by Ohshima et. al [24], who calculated the terminal velocity of a slowly sedimenting particle in the regime of $P e=0$ by considering a force-free particle at the steady state. In this approach, the total drag exerted on the particle is known and equal to the buoyancy force, which is the driving force behind the motion, and the terminal sedimentation velocity, $U_{\mathrm{SED}}$, is the unknown quantity in the problem. The study showed that in the presence of charged species such as ions in solution, the terminal velocity of a charged particle is smaller than the corresponding value in the absence of charges, $U_{\mathrm{SED}}^{\mathrm{ST}}$, obtained by writing a balance between buoyancy force and the Stokes' drag; i.e. $U_{\mathrm{SED}} / U_{\mathrm{SED}}^{\mathrm{ST}}<1$. The reciprocal of this problem is 


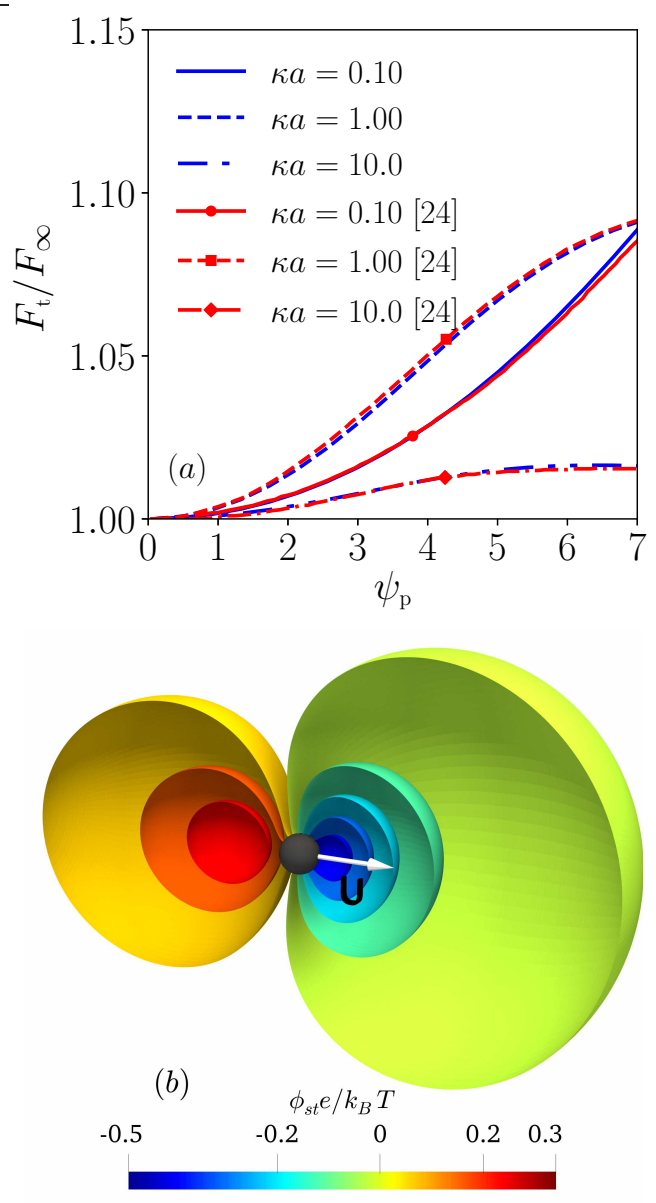

FIG. 2. Drag enhancement for a charged particle in unbounded fluid. (a) Calculation of the drag enhancement, $F_{\mathrm{t}} / F_{\infty}$, as a function of non-dimensional particle surface potential, $\psi_{\mathrm{p}}=\phi_{\mathrm{p}} e / k_{\mathrm{B}} T$. Curves marked with circle, square, and diamond represent the semi-analytical solution of Ohshima et. al [24] in the zero Pe regime. Calculations (blue curves) are presented for a particle of radius $a=50 \mathrm{~nm}$ in solutions of $\mathrm{KCl}$, where electrolyte properties are taken from Ref. [24]. These parameters include $T=298 \mathrm{~K}, \mu=0.891$ $\mathrm{mPa} \mathrm{s}^{-1}$, and $\epsilon_{\mathrm{r}}=78.5$. The non-dimensional parameters of the simulations are $\alpha=0.26, P e=0.2, \omega=0.96$. For $\kappa a=1$, and 10 , the results of computations converge to those of unbounded domain with an error of less than $1 \%$ considering $R / a=40$, and $L / a=80$ but for $\kappa a=0.1$, these parameters have been increased by a factor 4 in order to eliminate the boundary effects due to presence of the thick EDL. (b) Iso-surfaces of streaming potential, $\phi_{\text {st }}$, induced by the distortion of the EDL due to motion of the fluid. The streaming potential, $\phi_{\text {st }}$, corresponds to a case of $\kappa a=1$ and $\psi_{\mathrm{p}}=4$.

the case of a charged particle which moves with some constant velocity, $U$, in an electrolyte. In this case, the velocity, $U$, is known a priori and the resistive force due to the fluid, $F_{\mathrm{t}}$, is the unknown. From this perspective, the effect of electroviscous stresses leads to a resistive force larger than the Stokes's drag, $F_{\infty}$. In this study, we use the latter approach and show that both routes are in fact equivalent; i.e. $U_{\mathrm{SED}}^{\mathrm{ST}} / U_{\mathrm{SED}} \equiv F_{\mathrm{t}} / F_{\infty}$.

Figure 2a presents the results of our calculations of total drag, $F_{\mathrm{t}}$, including the contribution of electroviscous stresses for a system considered in Ref. [24] using constant surface potential, $\psi_{\mathrm{p}}=\phi_{\mathrm{p}} e / k_{\mathrm{B}} T$, on the particle surface. Noting that $U_{\mathrm{SED}}^{\mathrm{ST}} / U_{\mathrm{SED}} \equiv F_{\mathrm{t}} / F_{\infty}$, the results of our computation are in good agreement with the semi-analytical results of that study. It should be noted that in our simulations, the velocity $U$ is adjusted such that $P e$ is small enough and compatible with the underlying assumptions of the analytical solution of Ref. [24] $(P e \ll 1)$. Figure 2b presents iso-surfaces of streaming potential, $\phi_{\mathrm{st}}=\phi-\phi^{\mathrm{eq}}$, which may be thought of as the excess local electrical potential induced by the motion of the particle. As depicted, the convection of the charged species within the double layer polarizes the charge cloud giving rise to a dipolar streaming potential around the particle. Having validated the results of the theoretical model in a bulk electrolyte, we next provide a brief description of the experimental set-up and results. Following sections compare the results of our drag measurements with those of calculations.

\section{EXPERIMENTAL METHODS AND MATERIALS}

\section{A. Nanoslit preparation and experimental procedure.}

We fabricated silica slits of various heights using glass and Silicon/Silicon dioxide substrates as previously described [56]. First, an array of parallel channels was patterned on a Silicon dioxide surface using photolithography, and subsequently etched using Reactive-Ion Etching (RIE). Slit heights were measured using atomic force microscopy. Two different slit geometries were used for the experiments with heights of $2 h=261 \mathrm{~nm}$ and $2 h=397 \mathrm{~nm}$ and a width of $5 \mu \mathrm{m}$. Fully functional nanoslits were created by anodically bonding the processed Silicon/Silicon dioxide substrates with glass coverslips of thickness $\sim 170 \mu \mathrm{m}$, compatible with high NA microscopy. We loaded the device with a suspension of fluorescent particles at a number density of $2.7 \times 10^{8}$ $\mathrm{mL}^{-1}$ in a solution of salt concentration $0.1 \mathrm{mM}$ using a pressure driven flow through the slit. After a few minutes, the flow was arrested and the reservoir at the outlet of the slits was filled with the particle solution. The device was sealed in order to prevent evaporation and the system was allowed to equilibrate for $15 \mathrm{~min}$ before measurement. The electrical conductance of the particle suspension used was measured both before and after the experiment in order to determine the salt concentration during the measurement. For all experiments, the salt concentration was $0.1 \mathrm{mM}$ corresponding to a Debye length $\kappa^{-1} \approx 30 \mathrm{~nm}$. In order to examine the effect of ion size on particle diffusion, we also performed experiments with three different monovalent salts, namely $\mathrm{LiCl}, \mathrm{CsCl}$ 
and Tetrahexylammonium Chloride (THAC).

\section{B. Measurements of nanoparticle diffusion coefficients}

Carboxylated fluorescent polystyrene beads with a nominal radius of $55 \mathrm{~nm}$ were used (F8887, Lot \#1761661, Life Technologies) for all experiments. The average particle radius was determined by measuring translational diffusion using two-focus fluorescence correlation spectroscopy (2fFCS) and single particle tracking (SPT) in free solution. These measurements showed that the average particle radius is approximately $63 \mathrm{~nm}$, hence in our 3D-slit simulations, we use a particle radius of $a=63 \mathrm{~nm}$. The details of $2 \mathrm{fFCS}$ setup were similar to that described in Müller et. al [57]. Briefly, the distance between the two foci was calibrated against a diffusion standard, usually a fluorescence dye molecule that can be excited using the same wavelength as the particles. We used an aqueous solution of Rhodamine 6G dye molecules (J62315, Alfa Aesar) that has a diffusion coefficient of $(4.14 \pm 0.05) \times 10^{-6} \mathrm{~cm}^{2} \mathrm{~s}^{-1}$ at $25^{\circ} \mathrm{C}$. In 2fFCS, although changes in coverslip thickness, or refractive index mismatches may introduce optical aberrations and distort the shape of the molecular detection function of each focus, the distance between the two foci acts as an intrinsic ruler for the diffusion coefficient calculation and is robust to aberrations. Having determined this interfocus distance, we measure the diffusion coefficient of the fluorescent bead species in solution at a concentration of about $1 \mathrm{nM}$, both in pure water and in $10 \mathrm{mM} \mathrm{NaCl}$ solution. The values obtained from these measurements were used to determine the true radius of the particles in our experiments and the corresponding value of hydrodynamic drag in free solution, $F_{\infty}$.

Diffusion coefficient measurements on nanoparticles, confined in nanoslits of various heights and in free solution, were performed using wide-field fluorescence imaging and single particle tracking (SPT). In our custom built wide-field setup, fluorescence excitation was achieved by illuminating the particles with a $532 \mathrm{~nm}$ DPSS laser (MGL-III-532-100 mW, PhotonTec, Berlin) that was focused at the back aperture of a high numerical aperture objective (UPLSAPO 60×, 1.35 N.A., Olympus, UK). The emitted fluorescence was separated from the laser excitation using a dichroic mirror (F38-532, AHF Analysentechnik) and was imaged using a sCMOS camera (Prime 95B, Photometrics). Particle coordinates were obtained by fitting a 2D Gaussian function to the intensity profile in each image, and these coordinates were used to generate the particle trajectories. Diffusion coefficients were determined from plots of mean squared displacement vs. lag time [58].

\section{RESULTS}

\section{A. Axisymmetric simulations}

We began by investigating the role of the surface charge densities, $\sigma_{\mathrm{p}}$ and $\sigma_{\mathrm{w}}$, on the magnitude of the electroviscous effect. We varied both parameters independently and assessed their contribution to the total drag force, $F_{\mathrm{t}}$, exerted on a polystyrene particle of radius $a=50 \mathrm{~nm}$, where a mass density of $\rho \approx 1060 \mathrm{~kg} \mathrm{~m}^{-3}$ for polystyrene results in a ballistic velocity of $u_{\mathrm{s}} \approx 0.08 \mathrm{~m} \mathrm{~s}^{-1}$. First we considered an uncharged particle with $\sigma_{\mathrm{p}}=0$, traveling along the axis of a cylindrical pipe of surface charge density, $\sigma_{\mathrm{w}}$, in an electrolyte of $\kappa^{-1}=50 \mathrm{~nm}(\kappa a=1)$. Here, the electrolyte is a $\mathrm{KCl}$ solution whose properties were taken from Ref. [24], and therefore, the counterions correspond to $\mathrm{K}^{+}$with a Stokes radius of $r_{\mathrm{K}^{+}} \approx 1.25 \AA$ [48]. We determined the magnitude of the total drag force, $F_{\mathrm{t}}$, for various values of $R / a$ and $\sigma_{\mathrm{w}}$. To isolate the effect of electroviscous stresses, we define the electroviscous drag enhancement factor, $f_{\mathrm{EV}}=F_{\mathrm{t}} / F_{\mathrm{h}}$, as the ratio of total force on the confined particle to the pure hydrodynamic force in confinement and in the absence of any electrical charge in the system. Note that given the Stokes-Einstein relation, the enhancement factor is the same as the ratio $D_{\mathrm{h}} / D$ reported in Table I. In an unbounded fluid, $F_{\mathrm{h}}$ is equivalent to $F_{\infty}$ and therefore $f_{\mathrm{EV}}=F_{\mathrm{t}} / F_{\infty}$, which is the same as the quantity plotted in Fig. 2. We found that for our smallest value of $R / a=2, f_{\mathrm{EV}}$ is maximally $5 \%$ and occurred for relatively large wall charge densities, $S_{\mathrm{w}} \gtrsim 30$, which corresponds to $\sigma_{\mathrm{w}} \gtrsim 0.067 e \mathrm{~nm}^{-2}$ (Fig. 3a).

We then set $\sigma_{\mathrm{w}}=0$ and turned our attention to the role of electrical charge on the particle. We note that the drag enhancement factor, $f_{\mathrm{EV}}$, initially increases in response to increasing particle charge but then the rate of change of $f_{\mathrm{EV}}$ with respect to $S_{\mathrm{p}}$ decreases considerably (Fig. 3b). Our understanding of this behavior is linked to an increasing non-linearity in the electrostatics with increasing particle charge. For high values of surface charge, the Poisson-Boltzmann equation enters the highly non-linear regime characterized by over exponential decay in the electrical potential with distance from surface. In this regime, further increasing the surface charge only affects the volumetric charge density in the immediate vicinity of the surface and leaves the "farfield" nearly unchanged. The main contribution to the enhanced drag comes from the "far-field" of the particle, which for our purposes is the region of the electrolyte beyond a few nanometers from the particle surface. Due to the no-slip boundary condition at the particle surface, fluid velocities are very small in the near-field region which therefore does not make a substantial contribution to the drag force on the particle. Importantly, we note that confinement on its own can increase the magnitude of the electroviscous drag force compared to that in free solution (compare the solid and dashed curves with the dotted curves, Fig. 3b). Our interpretation of this obser- 

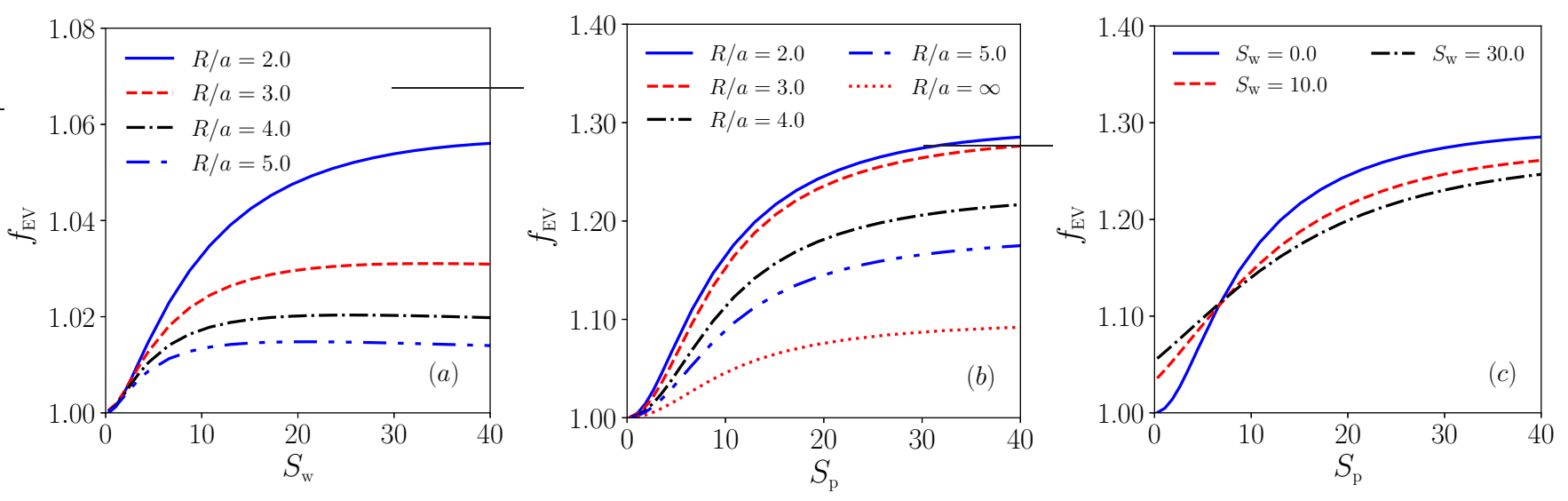

FIG. 3. Calculated electroviscous drag enhancement factor, $f_{\mathrm{EV}}$, for a polystyrene particle of radius $a=50 \mathrm{~nm}$ moving with velocity, $U=u_{\mathrm{s}} \approx 0.08 \mathrm{~m} \mathrm{~s}^{-1}$, along the axis of a pipe of radius, $R$. The electrolyte contains $\mathrm{KCl}$, and the Debye length, $\kappa^{-1}=50 \mathrm{~nm}$, with all parameters taken from Ref. [24]. These parameters include $T=298 \mathrm{~K}, \mu=0.891 \mathrm{mPa} \mathrm{s}^{-1}$, and $\epsilon_{\mathrm{r}}=78.5$. The non-dimensional parameters are $\alpha=0.26, P e=2.2, \omega=0.96, L / a=40, \kappa a=1$. (a) $f_{\mathrm{EV}}$ for an uncharged particle $\left(S_{\mathrm{p}}=0\right)$ moving along the axis of a tube of surface charge density, $S_{\mathrm{w}}$. (b) $f_{\mathrm{EV}}$ for motion of a charged particle in an uncharged pipe $\left(S_{\mathrm{w}}=0\right)$. (c) Effect of overlapping double layers on $f_{\mathrm{EV}}$ for the confined case of $R / a=2$, when both particle and wall are charged.

vation is that the main contribution of the confining walls to the observed drag enhancement is the spatial compression of the EDL around the particle. The amplification of the local charge density increases the effective polarizability of the EDL in fluid flow, which in turn enhances the drag force on the particle (see Fig. 2b). Figure 4 elucidates this point presenting a plot of the equilibrium charge density, $\rho_{\mathrm{e}}^{\mathrm{eq}}$, along a line parallel to the axis of the cylinder which passes through the gap between particle and wall. The local charge density around the particle, $\rho_{\mathrm{e}}^{\mathrm{eq}}$, clearly increases in response to confinement.

Next we examine the effect of overlapping EDLs for $R / a=2$ in situations where both particle and wall carry surface charges (Fig. 3c). Counterintuitively, we note that when particle is strongly charged, $f_{\mathrm{EV}}$ reduces slightly in response to an increase in magnitude of wall surface charge, $S_{\mathrm{w}}$. The examination of the equilibrium potential, $\phi^{\text {eq }}$, along the axis of pipe reveals that increasing the surface charge of the wall tends to shift the centerline potential, far away from particle, towards negative values (Fig. 5). This is a known effect in nanochannels with strongly overlapping EDLs where a shift in the centerline potential is required to satisfy the electroneutrality condition. The potential shift, in fact, elevates the number density of counterions, $n_{+}$, to values beyond the bulk density, $n_{\mathrm{b}}$, everywhere in the channel. In this situation, the parameter, $\kappa^{-1}$, does not serve as a good approximation for the actual thickness of the EDL, and attempts have been made to introduce an effective Debye length to address this problem [59,60]. Baldessari [60] showed that the effective thickness of the diffuse double layer in nanochannels with EDL overlap is given by $\kappa^{-1} / \sqrt{\cosh \left(\phi_{\mathrm{c}}^{\mathrm{eq}} e / k_{\mathrm{B}} T\right)}$, where $\phi_{\mathrm{c}}^{\mathrm{eq}}$ is the centerline potential in the channel. The effective Debye length therefore decreases with increasing departure of the center- line potential in the channel from zero. Hence, our understanding of the reduction in $f_{\mathrm{EV}}$ in response to an increase in $S_{\mathrm{w}}$ relates to the reduction of the effective thickness of EDL in the system. As shown in Fig. 5, far away from particle, the non-dimensional centerline potential, $\phi_{\mathrm{c}}^{\mathrm{eq}} e / k_{\mathrm{B}} T$, corresponding to a case with $S_{\mathrm{w}}=30$ is approximately -1.34 which is tantamount to $\approx 30 \%$ reduction in the EDL thickness.

This preliminary examination of the electroviscous effect in a simple cylindrical geometry reveals that the surface charge density in the system, particularly that of the particle, does exert an influence on the value of $f_{\mathrm{EV}}$. However, $f_{\mathrm{EV}}$ is proportional to particle surface charge only in the weakly charged limit and rapidly loses its sensitivity to surface charge when the electrostatics enters the highly non-linear regime. This indicates that the surface charge densities in the system are not likely to serve as suitable tuning parameters to explain the significantly higher drag enhancement factors reported in the experimental literature [33-37]. We further note the that charge on the confining walls is expected to play a relatively minor role in the confined electroviscous effect compared to the charge on the particle.

Next, we examine drag enhancement as a function of the parameter $\kappa a$ for varying values of the confinement parameter, $R / a$, and two values of particle radius, $a=5$ and $50 \mathrm{~nm}$. The trends presented in Fig. 6 reveal that the drag enhancement factor, $f_{\mathrm{EV}}$ depends on both the electrostatic system size, $\kappa a$, and the confinement parameter, $R / a$. Clearly, smaller values of both parameters promote an increase of the electroviscous drag. Furthermore, we find that an order of magnitude reduction in the radius of the particle dramatically lowers the drag enhancement factor, $f_{\mathrm{EV}}$. This is due to the fact that in our study, $U \propto u_{\mathrm{s}}$, and therefore, the Péclet number varies in pro- 

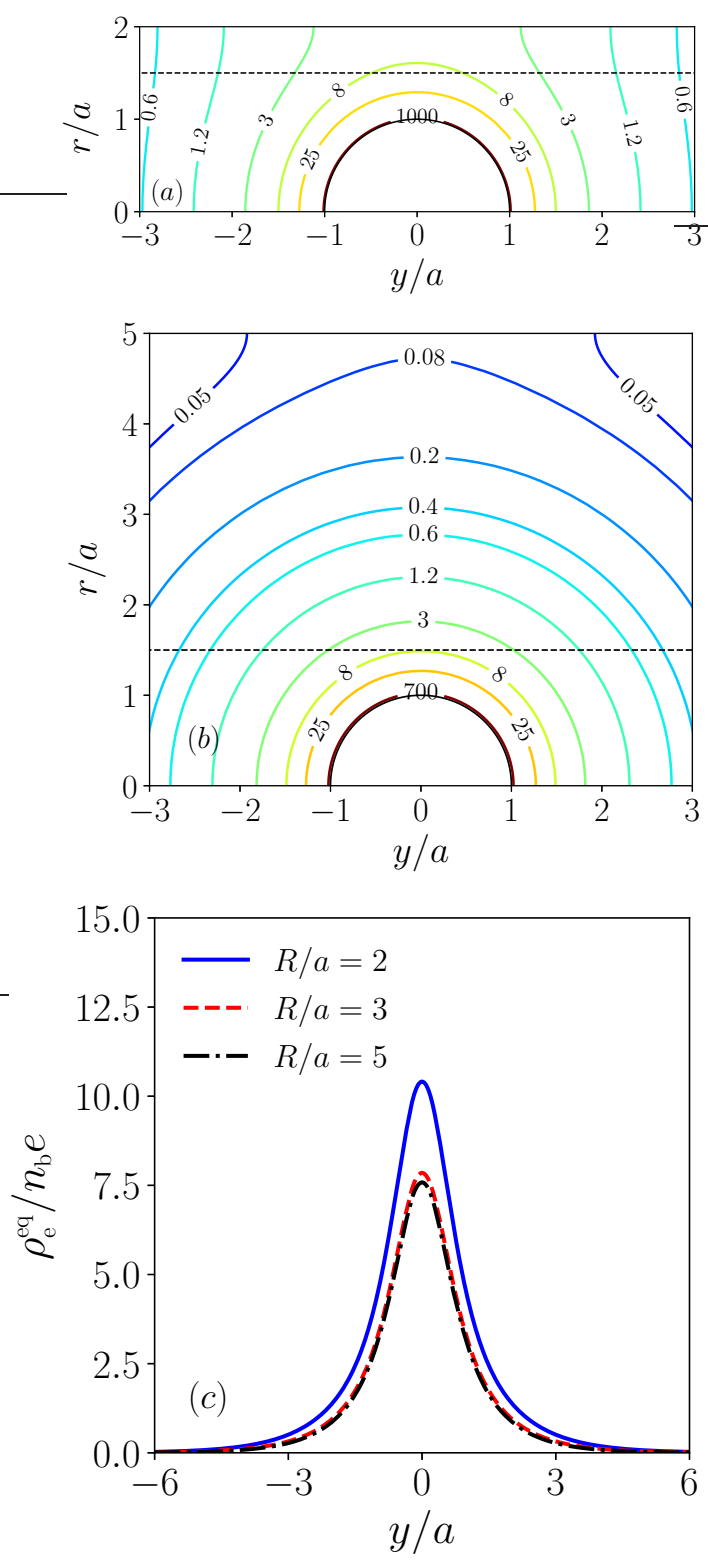

FIG. 4. Spatial distribution of charge density, $\rho_{\mathrm{e}}^{\mathrm{eq}} / n_{\mathrm{b}} e$, around a confined particle at equilibrium $\left(\kappa a=1, S_{\mathrm{w}}=0\right.$, $S_{\mathrm{p}}=45$ ) for (a) $R / a=2$, and (b) $R / a=5$. (c) Variations of the equilibrium charge density along a line parallel to the axis of the pipe located at $r / a=1.5$ (dashed lines in (a) and (b)). The charge density increases due to compression of the EDL by the confining wall.

portion to $a^{-1 / 2}$. Smaller particles imply a comparatively larger $P e$ and therefore the process of charge transport is dominated by convection. Large convective velocities can lead to very large distortions in the cloud, and ultimately, for very large $P e$, the EDL will be swept away by the fluid flow; i.e. the electroviscous effect vanishes as $P e \rightarrow \infty[26]$.

A related observation concerns the effect of particle mass. For a given particle size, an altered velocity of

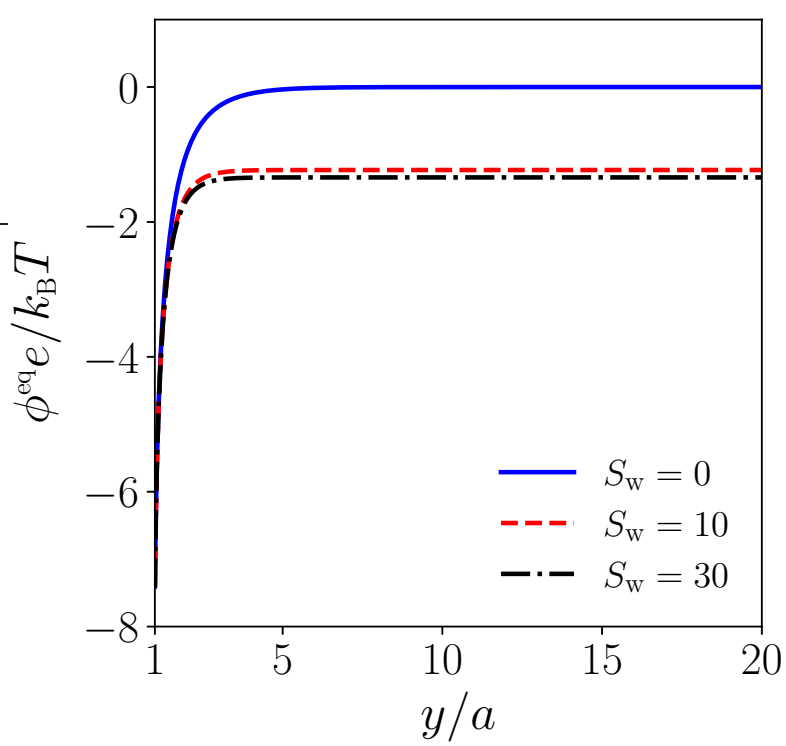

FIG. 5. Variation of the equilibrium electrical potential, $\phi^{\text {eq }}$, along the axis of the pipe as a function of wall charge density, $S_{\mathrm{w}}$. The curves correspond to a case with $\kappa a=1$, where $S_{\mathrm{p}}=45$. Far away from particle, the centerline potential deviates from zero as $S_{\mathrm{w}}$ increases, leading to smaller effective EDL thicknesses.

ballistic motion may be expected to influence the magnitude of electroviscous drag. Such a change in the velocity scale for a given radius could arise for particles of a different density. For example as $u_{\mathrm{s}} \sim \rho^{-1 / 2}$, gold particles with a density of approximately $19.3 \times 10^{3} \mathrm{~kg} \mathrm{~m}^{-3}$ would be described by a much lower average ballistic velocity compared to polystyrene particles with a density of approximately $1060 \mathrm{~kg} \mathrm{~m}^{-3}$; i.e., $U_{\mathrm{PS}} / U_{\mathrm{Au}} \approx 4$. We examine this contribution by altering $U$ in the calculation retaining all remaining parameters constant. Interestingly, we find that an order of magnitude reduction in particle velocity can indeed enhance $f_{\mathrm{EV}}$ to values up to about 1.5 for strongly confined, weakly screened particles of radius about $50 \mathrm{~nm}$ in a tube-like axisymmetric confinement. While the reduction of $P e$ leads to a considerable increase in $f_{\mathrm{EV}}$ for the axisymmetric case shown in Fig. 6a, we will show in the following sections that this effect is much smaller in a parallel plate slit which resembles most closely the experimental studies on gold nanoparticles $[33,36,37]$. Hence, the large hindered diffusion coefficients reported for gold particles cannot be attributed to a change in average velocity of the diffusing particles as a result of higher density of gold.

\section{B. 3D simulations}

In this section, we discuss the $3 \mathrm{D}$ simulations for system parameters corresponding to our experimental results. The axisymmetric simulations showed that surface charge associated with wall has only a small effect on the 

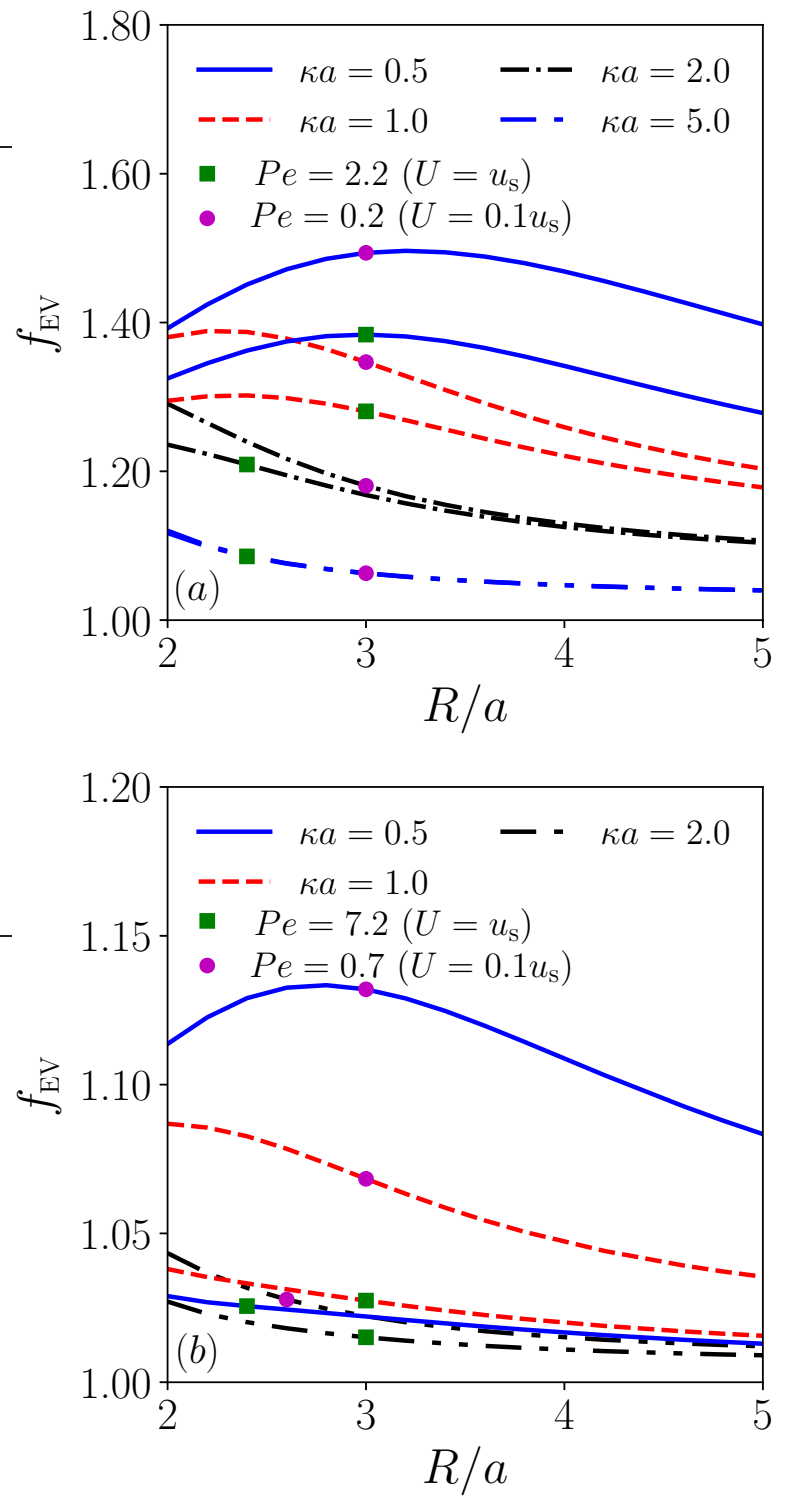

FIG. 6. Effect of EDL thickness on the electroviscous drag enhancement factor, $f_{\mathrm{EV}}$. (a) Calculated $f_{\mathrm{EV}}$ values for a polystyrene particle of radius, $a=50 \mathrm{~nm}$, traveling along an axisymmetric tube of radius, $R$, at two different particle velocities namely, $U=0.1 u_{\mathrm{s}}$ and $U=u_{\mathrm{s}} \approx 0.08 \mathrm{~m} \mathrm{~s}^{-1}$, and for various values of $\kappa a$. (b) Results of the same study as in (a) performed for a polystyrene particle of radius, $a=5$ $\left(u_{\mathrm{s}} \approx 2.7 \mathrm{~m} \mathrm{~s}^{-1} \mathrm{~nm}\right)$. All calculations were performed for $\mathrm{KCl}$ solutions, where corresponding non-dimensional parameters are $\alpha=0.26, \omega=0.96, S_{\mathrm{p}}=60, S_{\mathrm{w}}=0$, and $L / a=80$.

total drag exerted on a charged particle moving along the axis of a pipe. Our preliminary 3D simulations showed that the effect is even smaller for a $3 \mathrm{D}$ slit compared to its axisymmetric counterpart. We regarded the magnitude of the contribution as not significant enough to justify the computational cost of resolving the wall double layers throughout the slit geometry, and therefore set $S_{\mathrm{w}}=0$ for all calculations involving the slit geometry.
TABLE IV. Input parameters for 3D simulations corresponding to slit experiments. For all cases, bulk concentration of salt is fixed to $0.1 \mathrm{mM}$.

\begin{tabular}{cccccc}
\hline \hline $2 h(\mathrm{~nm})$ & $a(\mathrm{~nm})$ & $T(\mathrm{~K})$ & $\epsilon_{\mathrm{r}}$ & $\mu(\mathrm{mPa} \mathrm{s})$ & $u_{\mathrm{s}}\left(\mathrm{m} \mathrm{s}^{-1}\right)$ \\
\hline $261^{\mathrm{a}}$ & $63^{\mathrm{b}}$ & 295 & $78.5^{\mathrm{c}}$ & 0.954 & $0.06^{\mathrm{e}}$ \\
$397^{\mathrm{a}}$ & $63^{\mathrm{b}}$ & 295 & $78.5^{\mathrm{c}}$ & 0.954 & $0.06^{\mathrm{e}}$ \\
\hline
\end{tabular}

a Slit height measured by atomic force microscopy.

b Particle radius determined by free-diffusion measurements.

c $\epsilon_{0}=8.854 \times 10^{-12} \mathrm{~F} \mathrm{~m}^{-1}$.

d Ballistic velocity of the particle, $u_{\mathrm{s}}=\sqrt{k_{\mathrm{B}} T / m_{\mathrm{p}}}$, where mass of polystyrene particle $m_{\mathrm{p}}$ is calculated by considering a mass density of $\rho=1060 \mathrm{~kg} \mathrm{~m}^{-3}$.

We now examine the calculated drag enhancement for the experimental cases corresponding to a particle of radius $a=63 \mathrm{~nm}$ in parallel-plate slits of heights $2 h=261$, and $397 \mathrm{~nm}$, respectively. Simulations are performed using different electrolyte solutions ( $\mathrm{CsCl}, \mathrm{LiCl}$, and THAC) with a bulk concentration of $0.1 \mathrm{mM}$ which corresponds to a Debye length of $\kappa^{-1} \approx 30 \mathrm{~nm}$. The input parameters for the simulations are determined based on experimental conditions and are listed in Table IV. Figure 7 represents the results of these simulations, and compares the drag enhancement factor, $f_{\mathrm{EV}}$, for a confined particle with that for a particle under the same conditions in an unbounded fluid. We note that as expected the drag enhancement for $\kappa h=6.6$ is lower than that for $\kappa h=4.3$ for a given salt species in solution. This is in-line with the view described in Sec. VI A that the general effect of confining walls is to increase the polarizability of the charge cloud around the particle, or in different terms to enhance the "interaction cross-section" of the diffuse charge cloud with the hydrodynamic flow field.

Finally, we examine the effect of counterion radius on the electroviscous effect. Similar to the discussion on $P e$ in Sec. VIA, the rate of relaxation of the counterion cloud to a hydrodynamic perturbation determines the overall magnitude of the electroviscous drag. An increase in counterion size results in a larger $P e$ which in principle is expected to decrease the magnitude of $f_{\mathrm{EV}}$. Figure $8 \mathrm{a}$ shows the effect of $P e$ on $f_{\mathrm{EV}}$ for different electrolytes in unbounded domain. As shown, $f_{\mathrm{EV}}$ increases with decreasing $P e$ but asymptotes to its maximum value in the $P e \ll 1$ regime where the timescale of diffusion of ions, $a^{2} / D_{+}$, is much smaller than the advective timescale $a / u_{\mathrm{s}}$. But in the regime of $P e=O(1)$, typical for polystyrene nanoparticles in this study, $f_{\mathrm{EV}}$ in fact weakly depends on $P e$. Figure $8 \mathrm{~b}$ represents this effect for particles confined in a $3 \mathrm{D}$ slit by plotting $f_{\mathrm{EV}}$ vs. $h / a$ for different values of $\kappa a$. As shown, contrary to axisymmetric simulations, an order of magnitude of reduction in $P e$ has only a negligible impact on the drag enhancement factor, $f_{\mathrm{EV}}$, for a $3 \mathrm{D}$ slit. A decrease in $D_{+}$, however leads to an increase in $\alpha$, which is an "intrinsic" Péclet number defined by the velocity scale of electroos- 


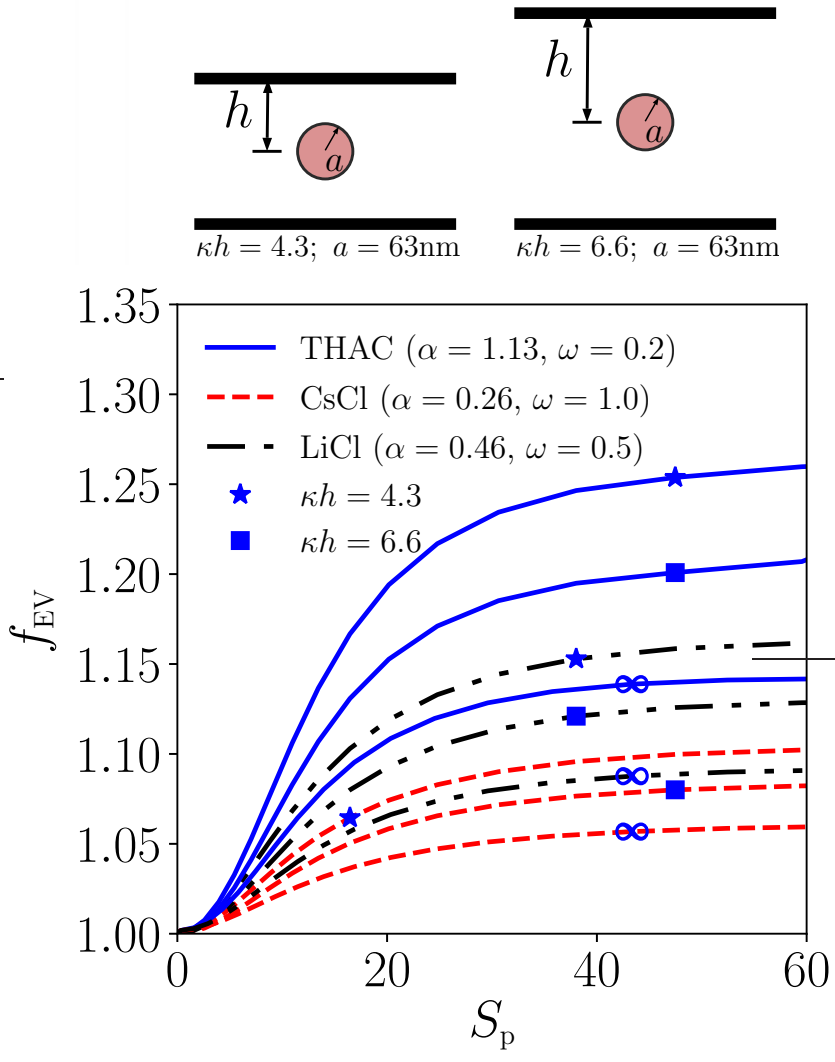

FIG. 7. Electroviscous drag enhancement factor, $f_{\mathrm{EV}}$, for a particle of radius, $a=63 \mathrm{~nm}$, moving along the mid-plane of a slit of height, $2 h=261,397 \mathrm{~nm}$. Symbols denote various geometrical configurations: $\kappa h=4.3$ (star), $\kappa h=6.6$ (square) and an unbounded fluid $(\infty)$. The confined cases of $\kappa h=$ 4.3 , and 6.6 are chosen specifically in accordance with the experimental configurations of this study. The Curves denote various counterion species: THAC (solid lines), CsCl (dashed lines) and $\mathrm{LiCl}$ (dashed-dotted lines). For all cases, $\kappa a=2.08$, $R_{\mathrm{d}} / a=20, S_{\mathrm{w}}=0$, and $U=u_{\mathrm{s}}$, which corresponds to a $P e=9.5,1.9$, and 3.8 for $\mathrm{THAC}, \mathrm{CsCl}$, and $\mathrm{LiCl}$, respectively.

motic flow, $\epsilon \zeta_{T}^{2} / \mu a$, in the system $[27,51]$. Since electroosmotic flow opposes the flow generated by the motion of particle, the overall result of an increase in counterion size is an enhancement of the electroviscous drag on the particle. Furthermore, although for low values of particle surface charge, the magnitude of electroviscous drag is expected to depend symmetrically on the size of both cations and anions in solution, for larger values of surface potential co-ions are effectively excluded from the EDL and therefore their contribution to drag enhancement becomes negligible [32]. Therefore, we focused our study on monovalent salts such that the co-ion species was chloride ion with a stokes radius of $r_{\mathrm{Cl}^{-}}=1.21 \AA$. Examining the influence of the cationic species in solution, we calculate $f_{\mathrm{EV}}$ for three counterion radii, namely $r_{\mathrm{Cs}^{+}}=1.19 \AA, r_{\mathrm{Li}^{+}}=2.39 \AA$ and $r_{\mathrm{THA}^{+}}=5.94 \AA$ representing $\mathrm{Cs}, \mathrm{Li}$ and THA cations in solution. We find a systematic increase in the asymptotic value of the drag enhancement factor, $f_{\mathrm{EV}}$, with increasing ion size. Over the range of cation radii and conditions probed, we note approximately $10-15 \%$ increase in $f_{\mathrm{EV}}$ for an increase in counterion radius by a factor $r_{\mathrm{THA}^{+}} / r_{\mathrm{Cs}^{+}}=5$.

Importantly, by comparing the axisymmetric results with 3D slit simulations, we note that the ratio of the total force in confinement, $F_{\mathrm{t}}$, to the confined hydrodynamic drag in the absence of any surface charge, $F_{\mathrm{h}}$, is in general larger for a charged particle in cylindrical tube or pipe-like confinement than for one in a parallel-plate slit. Our computational results suggest that depending on the
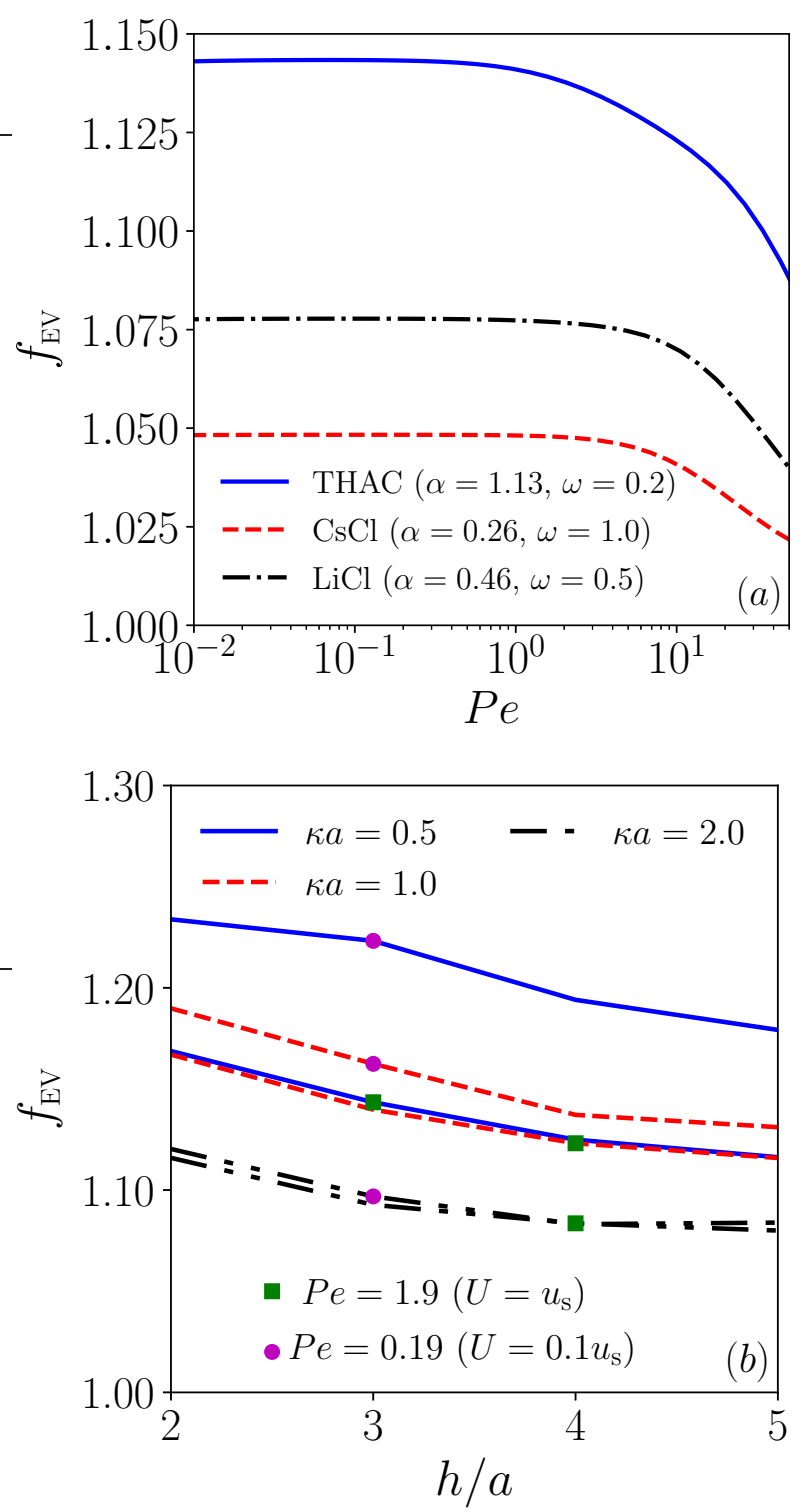

FIG. 8. Effect of $P e$ on drag enhancement factor, $f_{\mathrm{EV}}$. (a) $f_{\mathrm{EV}}$ for a particle of radius, $a=63 \mathrm{~nm}$, in an unbounded fluid, where $\kappa a=2.08$ and $S_{\mathrm{p}}=60\left(\sigma_{\mathrm{p}} \approx 0.1 e \mathrm{~nm}^{-2}\right)$. The electroviscous effect decreases significantly when $P e \gg 1$. (b) $f_{\mathrm{EV}}$ for the same particle as in (a) suspended in a solution of $\mathrm{CsCl}(\alpha=0.26, \omega=1.0)$ in a 3D slit. 
counterion species in the system, the drag enhancement factor, $f_{\mathrm{EV}}$, in a slit can be approximately $10-25 \%$ and $8-20 \%$ for configurations $\kappa h=4.3$ and $\kappa h=6.6$, respectively (Fig. 7).

\section{Comparison with experiments}

We now proceed to compare experimental measurements of the electroviscous effect with the values predicted by the computational model. The diffusion coefficients of the particles in bulk fluid, $D_{\infty}$, and under confinement, $D$, are two measurable quantities which can be used to relate experimental measurements to the calculated drag forces. In order to do so, we define an effective viscosity, $\mu_{\text {eff }}$, assuming that the total force, $F_{\mathrm{t}}$, on a confined particle can be represented by a modified Stokes law of the form, $F_{\mathrm{t}}=6 \pi \mu_{\mathrm{eff}} U a$. In other words, $\mu_{\mathrm{eff}}$ is an effective viscosity which represents the total contribution of hydrodynamic confinement, and electroviscous stresses to the drag force. Accordingly, the ratio of the total drag force on the confined particle, $F_{\mathrm{t}}$, to the Stokes drag on an uncharged particle, $F_{\infty}=6 \pi \mu U a$, can be seen as the ratio of the effective viscosity, $\mu_{\text {eff }}$, to the viscosity of the bulk fluid, $\mu$; i.e. $F_{\mathrm{t}} / F_{\infty}=\mu_{\text {eff }} / \mu$. It should be noted that $F_{\mathrm{t}} / F_{\infty}$ can also be written as $F_{\mathrm{t}} / F_{\mathrm{h}} \times F_{\mathrm{h}} / F_{\infty}$, where the first ratio, $F_{\mathrm{t}} / F_{\mathrm{h}}=f_{\mathrm{EV}}$, is regarded as the contribution due to electroviscous stresses alone, and the second, $F_{\mathrm{h}} / F_{\infty}=K_{\text {slit }}$, represents the hydrodynamic effect of the confining walls in the absence of the electrical charge in the system. According to fluctuation-dissipation theorem, this viscosity ratio relates directly to the experimental quantity, $D_{\infty} / D$, where $D$ is the diffusion coefficient measured for a confined particle and $D_{\infty}=k_{\mathrm{B}} T / 6 \pi \mu a$ is that measured for the same nanoparticle sample in free solution; i.e. $D_{\infty} / D=\mu_{\text {eff }} / \mu=K_{\text {slit }} \times f_{\mathrm{EV}}$. Figure 9 presents a comparison of experimental measurements and results of the computational model for $a=63$ $\mathrm{nm}$ and $\kappa^{-1} \approx 30 \mathrm{~nm}$, and for two values of system size $\kappa h=4.3,6.6$, and three different counterion species in solution. The ordinate on the left presents $\mu_{\mathrm{eff}} / \mu$ which reflects the ratio of diffusion coefficients, $D_{\infty} / D$, including the contributions of both hydrodynamic confinement and electroviscous forces. The ordinate on right presents the corresponding $f_{\mathrm{EV}}$ values which reflect contribution of the electroviscous stresses alone. As shown, for the geometries under consideration which are comparable to previous studies, $f_{\mathrm{EV}}$ is maximally $1.2-1.3$ and does not approach larger values $(\approx 2$ or larger $)$ that would explain the anomalously large hindered diffusion in previous studies [33-37]. In the experiments we also note a clear increase in the magnitude of the measured drag enhancement with increasing counterion radius. As expected, the effect is more pronounced for the $\kappa h=4.3$ case where the degree of confinement of the particle is higher.

Although calculations show that the magnitude of $f_{\mathrm{EV}}$ varies in proportion to the charge of the particle for low
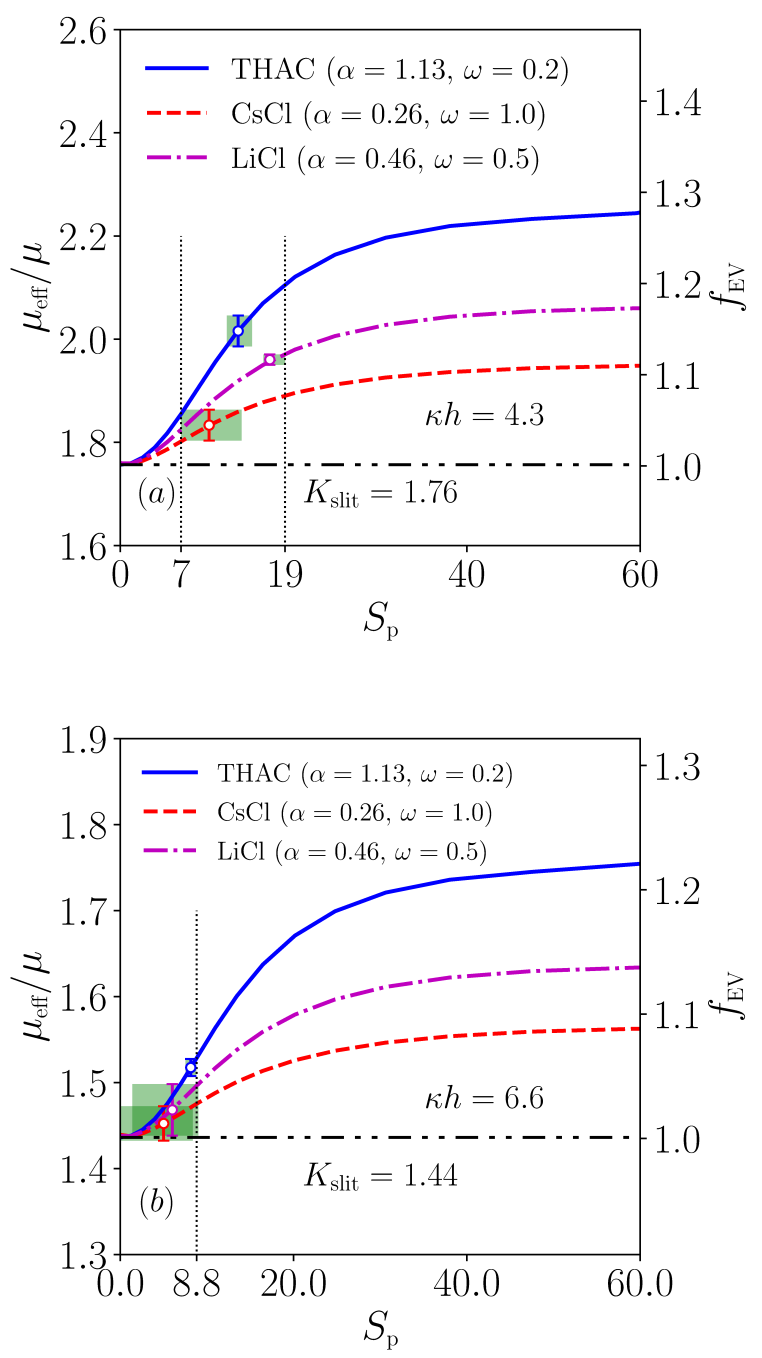

FIG. 9. Comparison of measured and calculated values of the effective viscosity, $\mu_{\mathrm{eff}}$, for a particle of radius $a=63 \mathrm{~nm}$ confined in a slit of height, $2 h$ for (a) $\kappa h=4.3(2 h=261$ $\mathrm{nm})$ and $(\mathrm{b}) \kappa h=6.6(2 h=397 \mathrm{~nm})$. The salt concentration is $0.1 \mathrm{mM}$ corresponding to a Debye length of $\kappa^{-1} \approx 30$ $\mathrm{nm}$. Open symbols correspond to the experimental data obtained from diffusion coefficient measurements of polystyrene nanospheres in nanoslits. Shaded zones (green) represent the range of possible values of particle surface charge density, $S_{\mathrm{p}}$, implied by the measured values and their corresponding uncertainties. For all cases, $\kappa a=2.08, R_{\mathrm{d}} / a=20, S_{\mathrm{w}}=0$, and $U=u_{\mathrm{s}}$, which corresponds to a $P e=9.5,1.9$, and 3.8 for THAC, $\mathrm{CsCl}$, and $\mathrm{LiCl}$, respectively. Horizontal lines illustrate the contribution of purely hydrodynamic confinement $\left(K_{\text {slit }}\right)$ to the effective viscosity.

values of particle charge density, the magnitude of the effective surface charge density of the particles in the experiment is a priori unknown and has not been directly measured. We therefore map the measured $\mu_{\text {eff }} / \mu$ values on to the computed curves and infer the range of particle surface charge implied by the measurement. We find the 
measurements lie on the curves within a range of particle surface charge density values of $S_{\mathrm{p}}=5-18$ which corresponds to charge densities of $\sigma_{\mathrm{p}}=0.009-0.0315 \mathrm{enm} \mathrm{nm}^{-2}$. Considering a charge regulation boundary condition [61] at the surface, this range corresponds to ionizable surface group densities of $0.01-0.1 \mathrm{~nm}^{-2}$ and is in good agreement with values typically encountered for polystyrene particles [62].

\section{CONCLUSION}

Polystyrene nanospheres suspended in a surfactantfree electrolyte containing well characterized monovalent ions at known concentrations, and confined in a parallelplate system composed of silica walls offers an ideal experimental system for a careful comparative study between experiment and theory for the electroviscous effect. We find that contrary to many previous reports, the experimental trends are well captured by the electrohydrodynamic model based on the PNPS system of equations. Calculations show that in general, depending on the geometry and electrolyte used in the system, the enhancement factor, $f_{\mathrm{EV}}$, is typically limited to about $1.1-1.25$ which is only about a $10 \%$ increase in drag compared to the bulk electroviscous effect [24]. Tube-like confinement is capable of generating much stronger ef- fects (20-30\% over the bulk electroviscous effect) but we have limited this study to parallel-plate systems which are the focus of several experimental studies in the literature [33-35, 37].

Our measurements on spheres in a parallel plate silica slit are in good agreement with the results of the standard electrohydrodynamic model and do not evince anomalously large values of drag enhancement factors, $f_{\mathrm{EV}}$, of upto a factor 2 reported in previous studies [3337]. Taken together, these results indicate that it is unlikely that previous reports of anomalously small diffusion coefficients for charged nanoparticles in confined fluidic systems can be attributed exclusively to an enhancement of the electroviscous effect. It is likely that measurements in the regime of weak electrostatic screening, where the electroviscous effect has a measurable effect, are disproportionately influenced by contributions from other sources such as confining potentials and surface inhomogeneities. It is also possible that organic or surfactant layers conferring surface charge on metallic nanoparticles or slit surfaces contribute to the effect in a manner that is not envisaged within the continuum electrohydrodynamic model based on PNPS equations. For experimental systems that do conform to the premises of the outlined theoretical model, we find that neither model nor measurement give any indication of anomalous enhancement of the electroviscous drag force on charged particles in confinement.
[1] W. M. Deen, AIChE Journal 33, 1409 (1987).

[2] R. Singh and J. W. Lillard, Exp. Mol. Pathol. 86, 215 (2009).

[3] B. Regner, D. Vučinić, C. Domnisoru, T. Bartol, M. Hetzer, D. Tartakovsky, and T. Sejnowski, Biophysical Journal 104, 1652 (2013).

[4] M. Baum, F. Erdel, M. Wachsmuth, and K. Rippe, Nature Communications 5, 4494 (2014).

[5] T. M. Squires and S. R. Quake, Rev. Mod. Phys. 77, 977 (2005).

[6] E. Beerdsen, D. Dubbeldam, and B. Smit, Phys. Rev. Lett. 96, 044501 (2006).

[7] M. S. Ray, Developments in Chemical Engineering and Mineral Processing 4, 254 (1996).

[8] H. Bruus, Theoretical microfluidics, Vol. 18 (Oxford university press Oxford, 2008).

[9] H. Faxen, Arkiv for Matematik, Astronomi och Fysik 17, 1 (1923).

[10] A. Goldman, R. Cox, and H. Brenner, Chem. Eng. Sci. 22, 637 (1967).

[11] Y. Pawar and J. L. Anderson, Industrial \& Engineering Chemistry Research 32, 743 (1993).

[12] M. E. O’Neill, Mathematika 11, 67-74 (1964).

[13] M. E. O'Neill, Mathematika 14, 170-172 (1967).

[14] N. Liron and S. Mochon, Journal of Engineering Mathematics 10, 287 (1976).

[15] J. W. Swan and J. F. Brady, Phys. Fluids 22, 103301 (2010).

[16] S. L. Dettmer, S. Pagliara, K. Misiunas, and U. F.
Keyser, Physical Review E 89, 062305 (2014).

[17] E. R. Dufresne, D. Altman, and D. G. Grier, Europhysics Letters (EPL) 53, 264 (2001).

[18] S. L. Eichmann and M. A. Bevan, Langmuir 26, 14409 (2010).

[19] M. Hosoda, K. Sakai, and K. Takagi, Physical Review E 58, 6275 (1998).

[20] L. Lobry and N. Ostrowsky, Physical Review B 53, 12050 (1996).

[21] J. H. Masliyah and S. Bhattacharjee, Electrokinetic and colloid transport phenomena (John Wiley \& Sons, 2006).

[22] F. Booth, Proc. Royal Soc. London A 203, 533 (1950).

[23] F. Booth, The Journal of Chemical Physics 22, 1956 (1954).

[24] H. Ohshima, T. W. Healy, L. R. White, and R. W. O'Brien, Journal of the Chemical Society, Faraday Transactions 2 80, 1299 (1984).

[25] R. G. Cox, Journal of Fluid Mechanics 338, 1 (1997).

[26] A. S. Khair, Langmuir 34, 876 (2018).

[27] D. A. Saville, Annual Review of Fluid Mechanics 9, 321 (1977).

[28] D. A. Saville, Advances in Colloid and Interface Science 16, 267 (1982).

[29] T. Li, S. Kheifets, D. Medellin, and M. G. Raizen, Science 328, 1673 (2010).

[30] D. McQuarrie, Statistical Mechanics (University Science Books, 2000).

[31] M. D. Graham, Microhydrodynamics, Brownian Motion, and Complex Fluids, 1st ed. (Cambridge University 
Press, 2018).

[32] G. A. Schumacher and T. G. M. van de Ven, Faraday Discussions of the Chemical Society 83, 75 (1987).

[33] S. L. Eichmann, S. G. Anekal, and M. A. Bevan, Langmuir 24, 714 (2008).

[34] N. Kaji, R. Ogawa, A. Oki, Y. Horiike, M. Tokeshi, and Y. Baba, Analytical and Bioanalytical Chemistry 386, 759 (2006).

[35] H. T. Hoang, I. M. Segers-Nolten, N. R. Tas, J. W. van Honschoten, V. Subramaniam, and M. C. Elwenspoek, Nanotechnology 22, 275201 (2011).

[36] N. Mojarad and M. Krishnan, Nature Nanotechnology 7, 448 (2012).

[37] S. Fringes, F. Holzner, and A. W. Knoll, Beilstein Journal of Nanotechnology 9, 301 (2018).

[38] Y. Kazoe, K. Mawatari, and T. Kitamori, Analytical Chemistry 87, 4087 (2015).

[39] R. Kubo, Reports on Progress in Physics 29, 255 (1966).

[40] B. Lin, J. Yu, and S. A. Rice, Physical Review E 62, 3909 (2000).

[41] J. Happel and H. Brenner, Low Reynolds number hydrodynamics: with special applications to particulate media, Vol. 1 (Springer Science \& Business Media, 2012).

[42] T. Bohlin, On the drag on a rigid sphere moving in a viscous liquid inside a cylindrical tube, Vol. 155 (Elander Stockholm, Sweden, 1960).

[43] S. Tabatabaei, T. van de Ven, and A. Rey, Journal of Colloid and Interface Science 301, 291 (2006).

[44] S. M. Tabatabaei and T. G. M. Van De Ven, Journal of Fluid Mechanics 656, 360 (2010).

[45] J. D. Sherwood, Physics of Fluids 21, 013101 (2009).

[46] K. Misiunas, S. Pagliara, E. Lauga, J. R. Lister, and U. F. Keyser, Physical Review Letters 115, 038301 (2015).

[47] W. B. Russel, J. Colloid Interf. Sci. 55, 590 (1976).

[48] P. C. F. Pau, J. O. Berg, and W. G. McMillan, The
Journal of Physical Chemistry 94, 2671 (1990).

[49] D. Lide, CRC Handbook of Chemistry and Physics: A Ready-reference Book of Chemical and Physical Data (CRC-Press, 1995).

[50] H. P. Langtangen, "Programming of finite element solvers," in Computational Partial Differential Equations: Numerical Methods and Diffpack Programming (Springer Berlin Heidelberg, Berlin, Heidelberg, 2003) pp. 251-458.

[51] E. Yariv, O. Schnitzer, and I. Frankel, Journal of Fluid Mechanics 685, 306 (2011).

[52] M. S. Alnæs, J. Blechta, J. Hake, A. Johansson, B. Kehlet, A. Logg, C. Richardson, J. Ring, M. E. Rognes, and G. N. Wells, Archive of Numerical Software 3 (2015), 10.11588/ans.2015.100.20553.

[53] G. Mitscha-Baude, A. Buttinger-Kreuzhuber, G. Tulzer, and C. Heitzinger, Journal of Computational Physics 338, 452 (2017).

[54] G. K. Batchelor, "Flow of a uniform incompressible viscous fluid," in An Introduction to Fluid Dynamics, Cambridge Mathematical Library (Cambridge University Press, 2000) p. 174-263.

[55] J. D. Jackson, Classical electrodynamics, 3rd ed. (Wiley, New York, 1999).

[56] M. Krishnan, N. Mojarad, P. Kukura, and V. Sandoghdar, Nature 467, 692 (2010).

[57] C. Müller, A. Loman, V. Pacheco, F. Koberling, D. Willbold, W. Richtering, and J. Enderlein, EPL (Europhysics Letters) 83, 46001 (2008).

[58] T. Savin and P. S. Doyle, Phys. Rev. E 71, 041106 (2005).

[59] F. Tessier and G. W. Slater, ELECTROPHORESIS 27, 686 (2006).

[60] F. Baldessari, Journal of Colloid and Interface Science 325, 526 (2008).

[61] M. Krishnan, The Journal of Chemical Physics 146, 205101 (2017).

[62] G. M. Kepler and S. Fraden, Physical Review Letters 73, 356 (1994). 